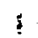

CHEMICAL ANAIYSIS OF SURFACES USING ALPHA PARTICLES

\author{
by \\ James H. Patterson \\ Chemistry Division \\ Argonne National Laboratory \\ Argonne, Illinois \\ Anthony L. Turkevich \\ The Enrico Fermi Institute for Nuclear Studies \\ University of Chicago \\ Chicago, Illinois \\ Ernest Franzgrote \\ Jet Propulsion Laboratory \\ Pasadena, California
}

\author{
Submitted to \\ The Journal of Geophysical Research \\ July 1964
}




\title{
CHEMICAL ANALYSIS OF SURFACES USING ALPHA PARTICLES
}

\author{
James H. Patterson* \\ Chemistry Division \\ Argonne National Laboratory \\ Argonne, Illinois \\ Anthony I. Turkevich** \\ The Enrico Fermi Institute for Nuclear Studies \\ University of Chicago \\ Chicago, Illinois \\ Ernest Franzgrote \\ Jet Propulsion Laboratory \\ Pasadena, California
}

\begin{abstract}
The chemical analysis of surfaces using alpha particle interactions has been investigated. Simple instruments incorporating $\mathrm{Cm}^{242}$ alpha sources and semiconductor silicon detectors are described. The characteristic energy spectra of alpha particles scattered at large angles from thick targets of different elements have been determined. The relative intensities of scattering by different elements have been established. They show large enhancement over Rutherford scattering for elements lighter than sodium due to nuclear effects. The production of protons from $(\boldsymbol{\alpha}, \mathrm{p})$ reactions in certain elements increases the sensitivity of the method for these elements. The theoretical basis for the quantitative interpretation of scattering spectra from complex materials is developed. The technique appears to be suitable for instrumented space missions.
\end{abstract}




\section{INTRODUCTION}

The usefulness of alpha particles for the chemical analysis of surfaces, originally suggested to one of the authors by Professor S. K. Allison of The Enrico Fermi Institute for Nuclear Studies, was explored in a preliminary report by Anthony Turkevich (Anthony Turkevich, 1961). In this article experimental data were presented showing that the energy spectra of backward scattered alpha particles were characteristic of the elements present in the scattering material. It was suggested that, based on these ideas, a rugged and compact analytical instrument could be designed using alpha radioactive nuclides as sources and semiconductor detectors, together with transistorized pulse height analyzers, as energy sensitive detectors. Such an instrument might be suitable for obtaining the gross chemical composition of a surface under conditions such as are being considered for instrumented lunar missions. There has been independent (Eskind and Mark, 1962) confirmation of some of these characteristics of alpha particle scattering.

Since the submission of the first report, additional work has been done on the potentialities of this technique. The spectra from a large number of elements have been obtained. The sensitivities for different elements have been determined. The matrix dependence has been studied. Finally, the usefulness of $(\alpha, p)$ reactions to increase the sensitivity for certain elements 
has been established. A start has been made on the development of calculational techniques needed to obtain quantitative results from complex mixtures.

A breadboard instrument incorporating these ideas and having close to possible flight geometries has been constructed and has operated successfully for about a year. This communication presents data obtained in the fundamental studies on the method,. describes the breadboard instrument, and presents some of the results obtained with it during the period 1960-1962.

\section{DESCRIPTION OF THE APPARATUS}

The general experimental arrangement has already been described (Anthony Turkevich, 1961). It consists of a source of alpha particles collimated so that a beam strikes the surface to be analyzed. One or more semiconductor detectors are placed close to the source so as to detect backward scattered alpha particles. Protons can be detected by similar detectors operated in such a way that the sensitive depth in the silicon is suitable for the longer range of these particles. Also, appropriately thin absorbers in front of these proton detectors remove alpha particles without significantly reducing the intensity of the protons. 


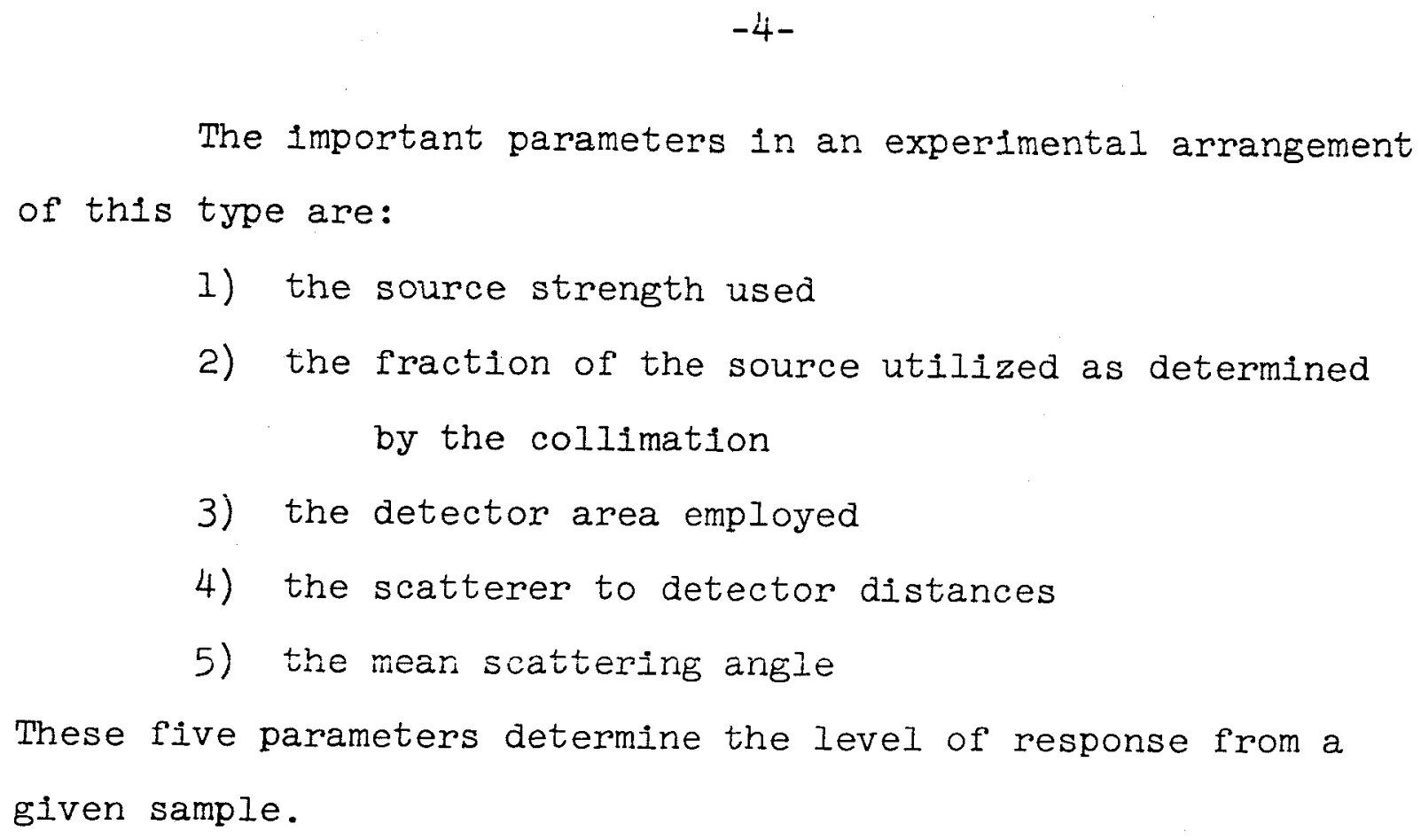

The important parameters in an experimental arrangement of this type are:

1) the source strength used

2) the fraction of the source utilized as determined by the collimation

3) the detector area employed

4) the scatterer to detector distances

5) the mear scattering angle

These five parameters determine the level of response from a given sample.

The data presented in this report were obtained primarily with three different systems, all designed to examine prepared samples. The first was a research apparatus constructed in duplicate. One of the units was used at the Argonne National Laboratory; the other at The Enrico Fermi Institute. It had only one detector position, but this could be made to be either an alpha or a proton detector.

The second apparatus was the "breadboard" instrument. It was designed to demonstrate that an instrument with close to the (then) flight requirements could be constmucted. It had one alpha detector and four proton detectors, and provision for four alpha sources.

A third system, called the "prototype", had the same number of source and detector units as the breadboard, but shorter 
scattering distances. This unit was not developed fully because changes in flight requirements occurred. Some data from this instrument, particularly as regards proton response, are included in this report.

The significant parameters of these three instmuments are summarized in Table I. Fig. I gives some construction details of the research apparatus; Fig. 2 has similar details for the breadboard instrument. All instruments were operated in vacuum with a special air lock for introducing samples.

The sources used in this work were $\mathrm{cm}^{242}$ ( $t \frac{1}{2}=163 \mathrm{~d}$, $\left.\mathrm{T}_{\boldsymbol{\alpha}}=6.11 \mathrm{Mev}\right)$. They were prepared from neutron irradiated $\mathrm{Am}^{241}$ by purification and then electrodeposition on to platinum discs. Recent sources have been prepared by evaporation on to stainless steel. The platinum discs were inserted into source holders that provided the collimation needed. When first prepared, the sources were very monoenergetic. Except in the case of the strongest sources, the full width at half maximum was less than two percent. With time, due to decay, the sources accumulated increasing amounts of $\mathrm{Pu}^{238}$ ( $t \frac{1}{2}=89 \mathrm{yr}$., $\left.\mathrm{T}_{\alpha}=5.49 \mathrm{Mev}\right)$. Also, any $\mathrm{Cm}^{244}$ ( $\left.\mathrm{t} \frac{1}{2}=17.6 \mathrm{yr}, \mathrm{T}_{\alpha}=5.8 \mathrm{Mev}\right)$ originally present, became relatively more important.

The detectors used in this work were surface barrier type silicon detectors. They were obtained either from ORTEC 


\section{FIGURE 1}

Research Apparatus used at the Argonne National Laboratory and The Enrico Fermi Institute for Nuclear Studies of The University of Chicago to study alpha particle scattering. Two perpendicular sections are shown.

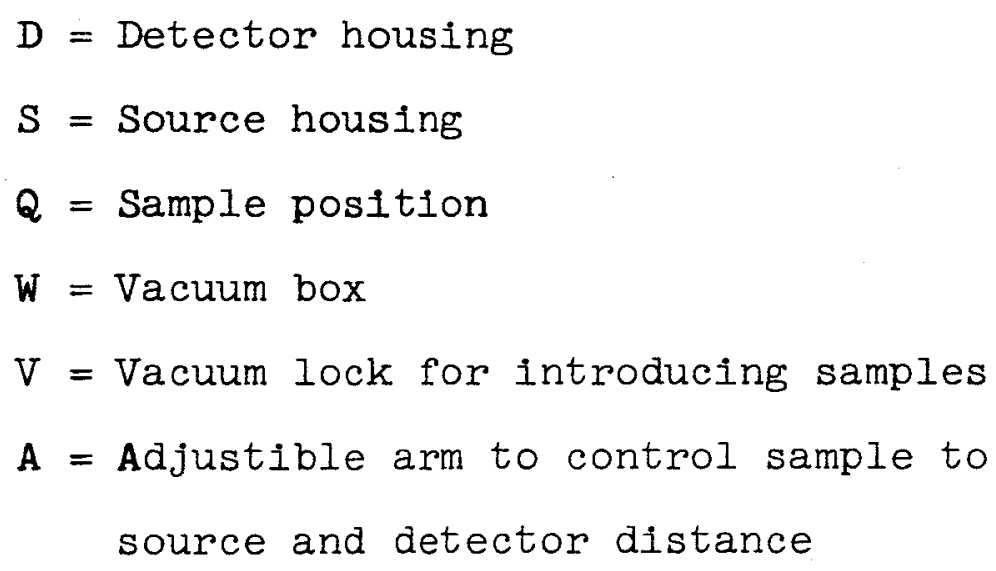



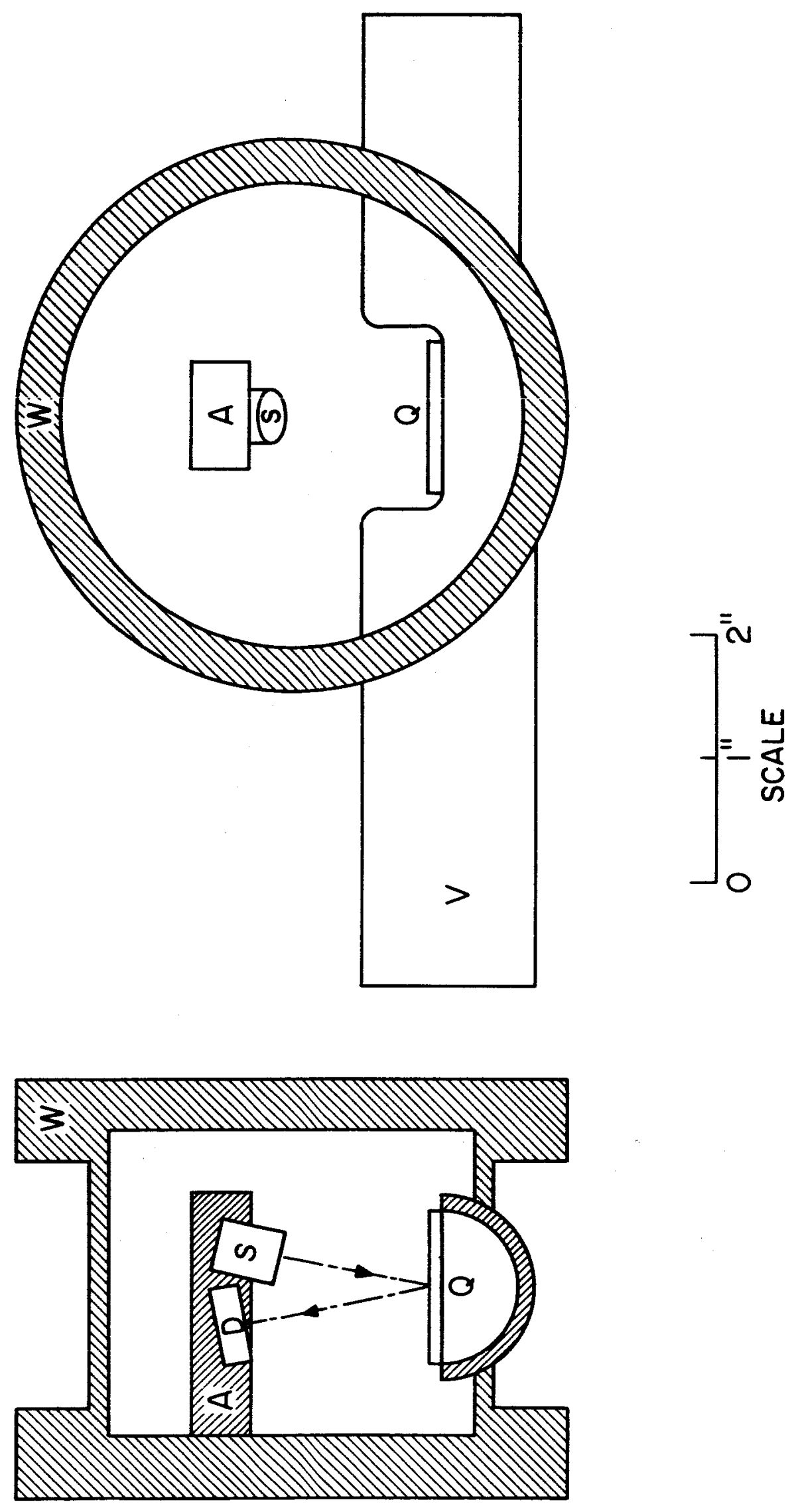


\section{FIGURE 2}

Construction Details of "Breadboard" Instrument for Chemical Analysis using Alpha Particle Interactions:

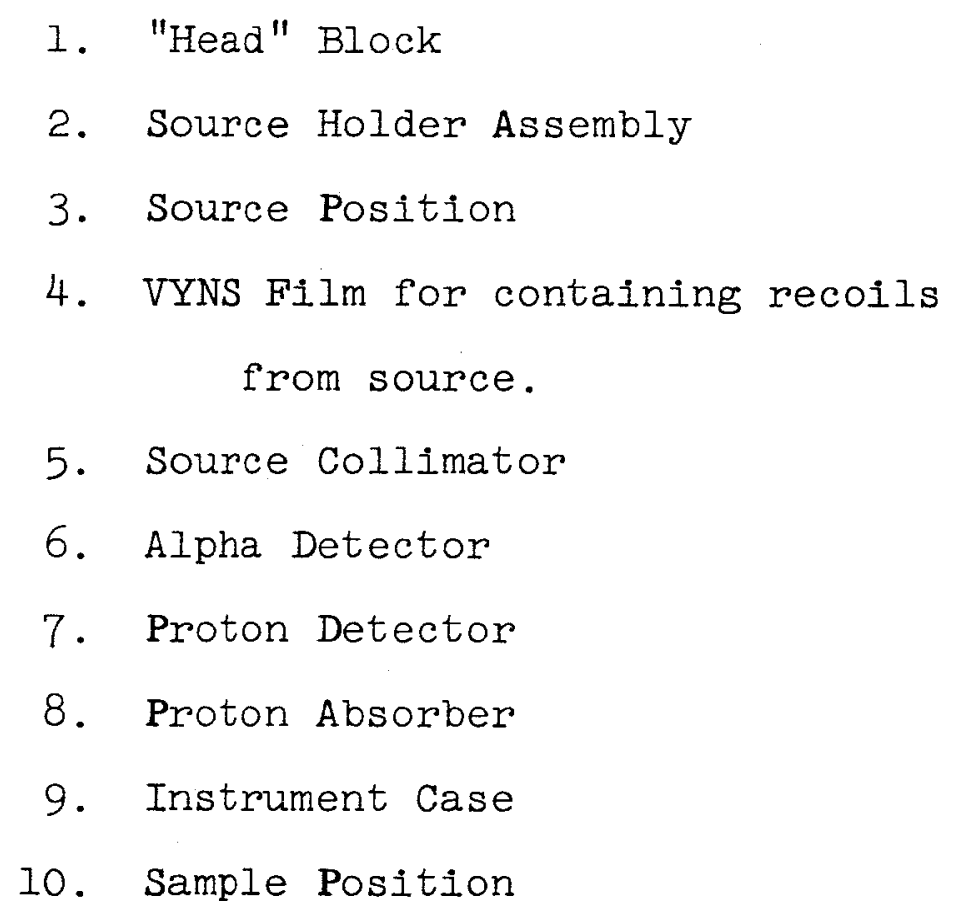

The breadboard instrument, including case and transistorized amplifiers and mixing circuit, weighed about $0.6 \mathrm{lbs}$. 


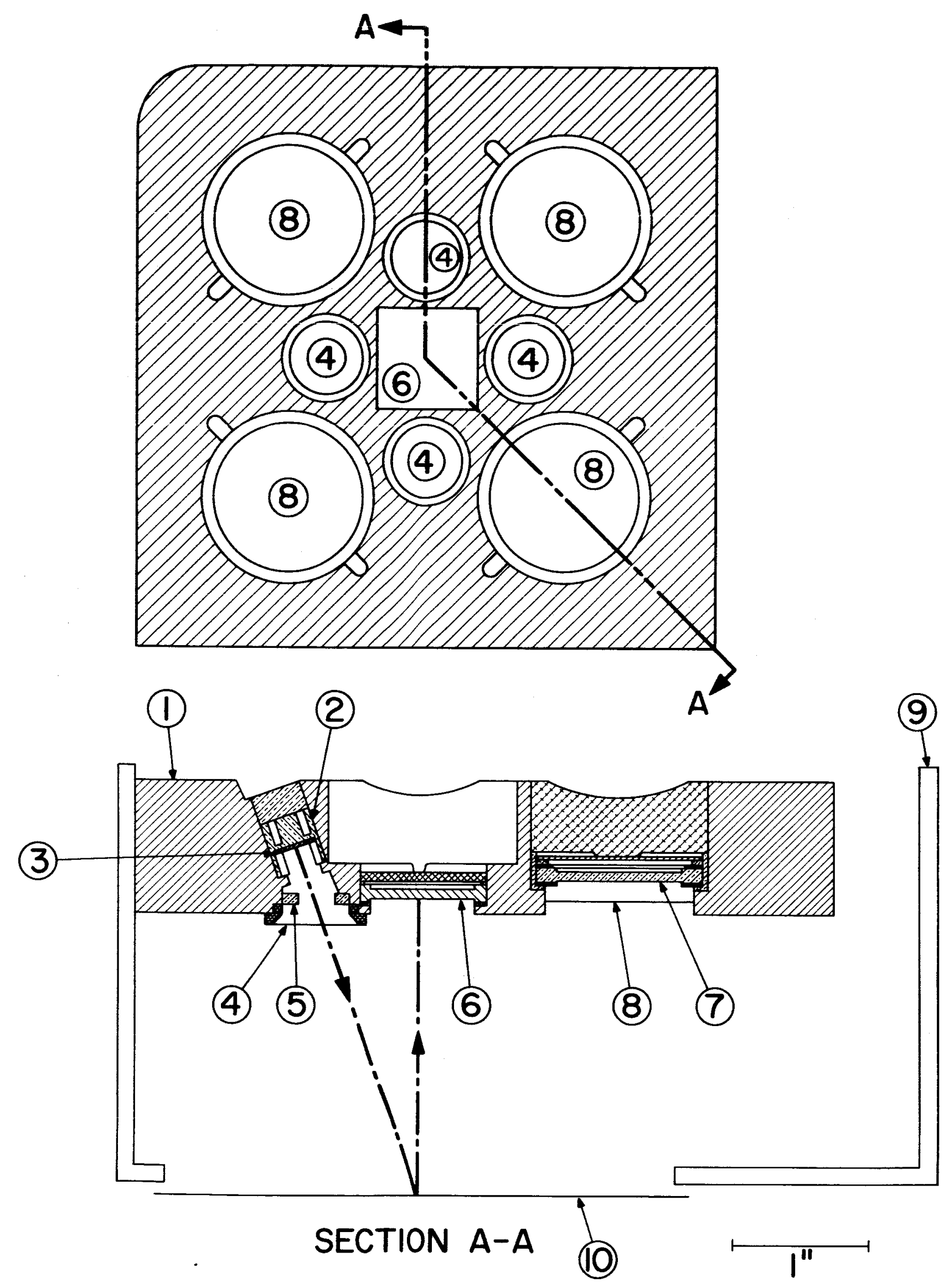


Parameters of Instruments Used to Study Chemical Analysis by Means of Alpha Particle Scattering

Research Breadboard Prototype Apparatus Instrument Instrument

Sources: Number

Total Strength, $\max (\mathrm{mc})^{*}$

Approximate Fraction Utilized

Distance to Sample $(\mathrm{cm})$

Area of Sample Examined $\left(\mathrm{cm}^{2}\right)$
1

8.0

0.012

4.13

8.0

1

0.49

Active Area $\left(\mathrm{cm}^{2}\right)$

Central Distance from Sample (cm) 4.13

Mean Scattering Angle

Proton Detectors: Number

Total Active Area $\left(\mathrm{cm}^{2}\right)$ $160^{\circ}$

Alpha Detectors: Number

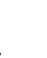

$$
4
$$

4

$$
5.0
$$

0.64

4

0.025

0.030

3.47

1.55

16.0

6.4

1 1

Central Distance from Sample $(\mathrm{cm}) 4.13^{* *}$

$0.49 * * \quad 4.0$

1.0

0.25

Mean Angle for $(\alpha, p)$ Protons

$$
160^{\circ} * *
$$

3.7

1.8

3.05

1.45

$160^{\circ}$

$160^{\circ}$

* This lists the maximum source strength used. Sometimes not all the sources were available, and with time, there was significant decay of the $\mathrm{Cm}^{242}$.

* The Alpha detector was replaced by a Proton detector in the Research Apparatus in the studies at the University of Chicago. 
at Oak Ridge, Tennessee, or from the Chicago Iaboratories for Applied Science. The detectors and the reverse biases applied were chosen to give depletion layers appropriate for the detection of alpha particles ( 50 microns) or protons (200-300 microns).

The electronics consisted, firstly, of transistorized charge sensitive amplifiers (except in the case of the Argonne National Laboratory Research Instrument). These amplifiers were inside the vacuum system in the case of the breadboard and prototype instruments. In the research instrument at the Argonne National Laboratory, a tube type voltage sensitive preamplifier was used. The output of the amplifiers was fed, through an additional amplifier, into a Nuclear Data pulse height analyzer. The stability of the detection system was checked frequently with monochromatic sources. During the long times needed in much of this work, the stability was monitored by the small amounts of contamination present in the instrument. Changes in amplification of more than one percent during a run were very rare. The detection system was linear in energy response to better than five percent. The samples examined were either flat pieces of metal or powders presented in trays through the vacuum lock. The distance of the sample from the source and detector was usually controlled to about half a millimeter. 


\section{EXPERIMENTAL RESULTS}

The research instrument was used primarily to establish the characteristics of this type of analysis -- the shapes of the spectra from different elements, the intensities of scattering, checks on intensity ratios in compounds, etc. The breadboard instrument demonstrated the feasibility of constructing a flight suitable instrument based on these ideas. It also provided much fundamental information on the method.

The shapes of spectra from thick samples of elements heavy enough to scatter alpha particles primarily by Rutherford scattering are expected to be essentially rectangular in shape with a high energy endpoint determined by the mass of the scatterer (Turkevich, 1961). Fig. 3 illustrates this behavior with scattering curves from aluminum, silicon, titanium, and iron obtained with the research apparatus. Fig. 3b shows corresponding scattering curves from boron, graphite, chromium, silver, and platinum. Fig. 3c shows data for Beryllium, sulfur, copper and zirconium. In each case the scattered intensity is plotted on a logarithmic scale as a function of the fraction of the incident kinetic energy that remains in the scattered alpha particle, $T / T_{0}$. The theoretical high energy endpoints for each element, (Turkevich, 1961) is indicated by an arrow.

As examples of scattering spectra from some simple compounds, Fig. 4 presents the spectra of alpha particles 


\section{FIGURE 3}

Alpha scattering spectrums from thick targets of some pure elements measured with the Research Apparatus. The unit of the abscissas is the fraction of the incident energy, $T / T_{0}$, remaining with the scattered alpha particle. The ordinates are intensities of the scattered alpha particles in units of counts per $100 \mathrm{~min}$, per $10^{10} \mathrm{~d} / \mathrm{m}$ of $\mathrm{cm}^{242}$ in the alpha source, per four channels of the analyzer (approximately 0.02 in $\left.\mathrm{T} / \mathrm{T}_{0}\right)$.
a. Aluminum, silicon, titanium, iron
b. Boron, graphite, chromium, silver, platinum

c. Beryllium, sulfur, copper, zirconium

The arrows show, for each element, the predicted high energy cut-off according to Equation 1 of the discussion section. 


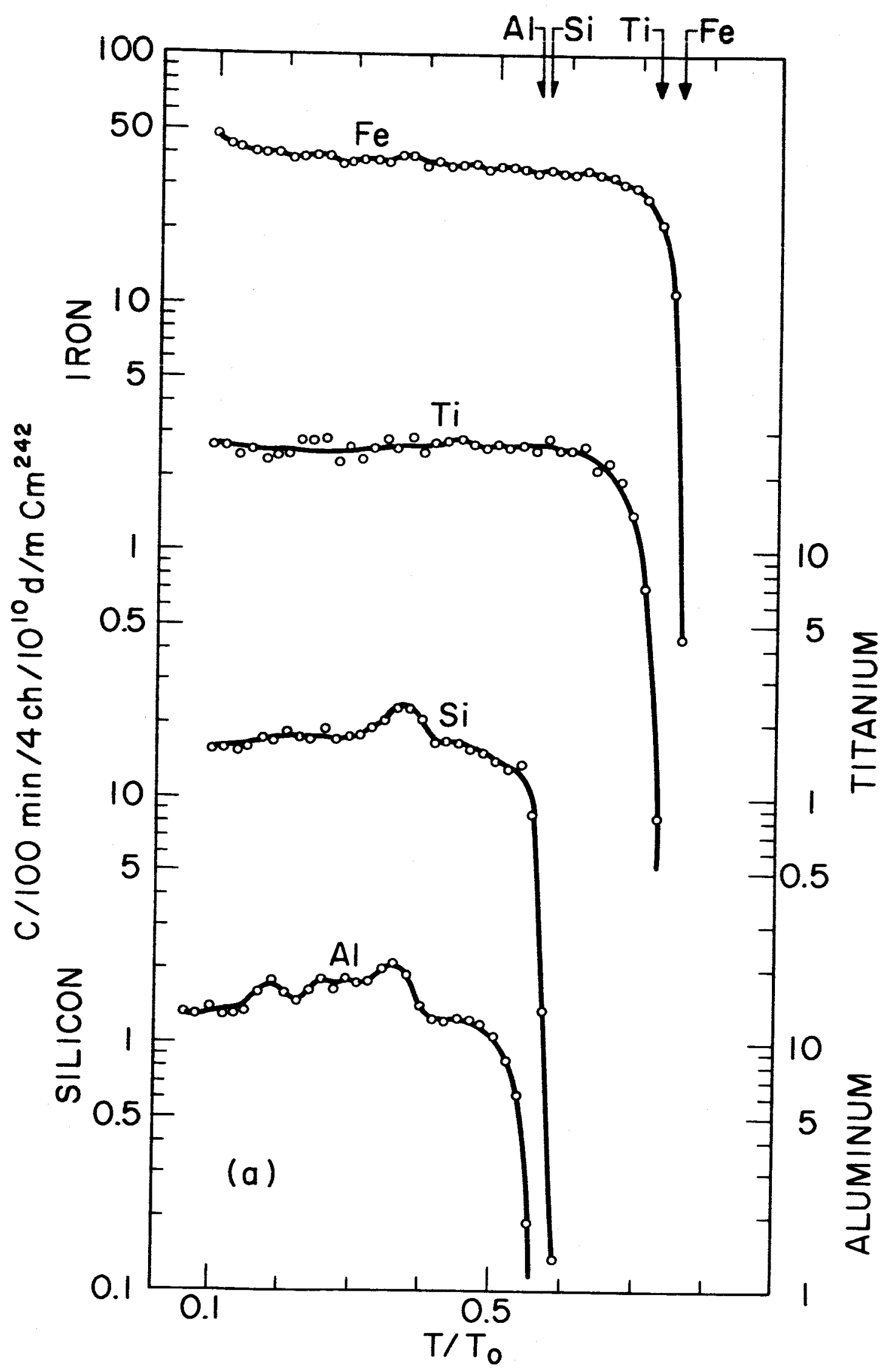




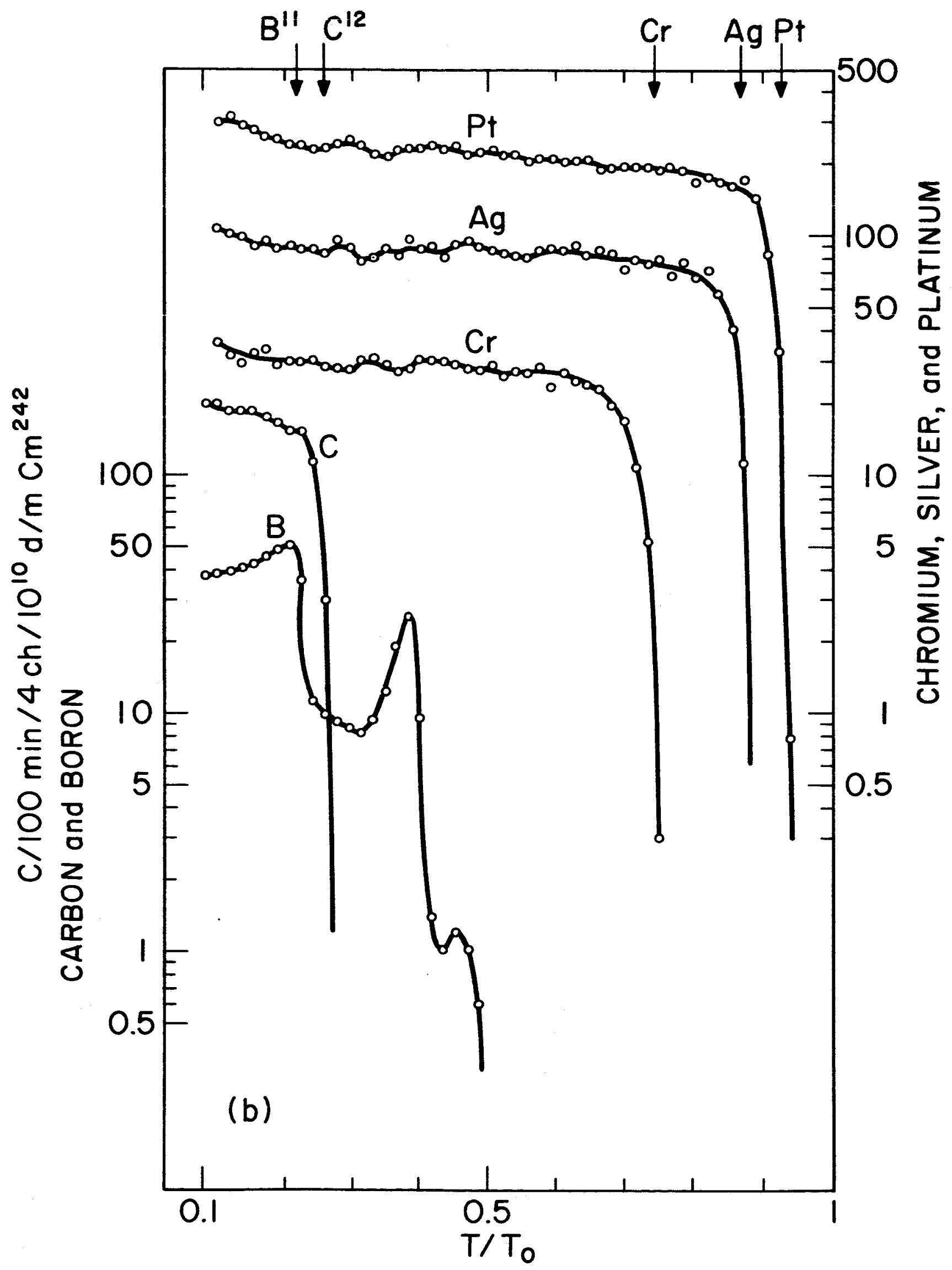




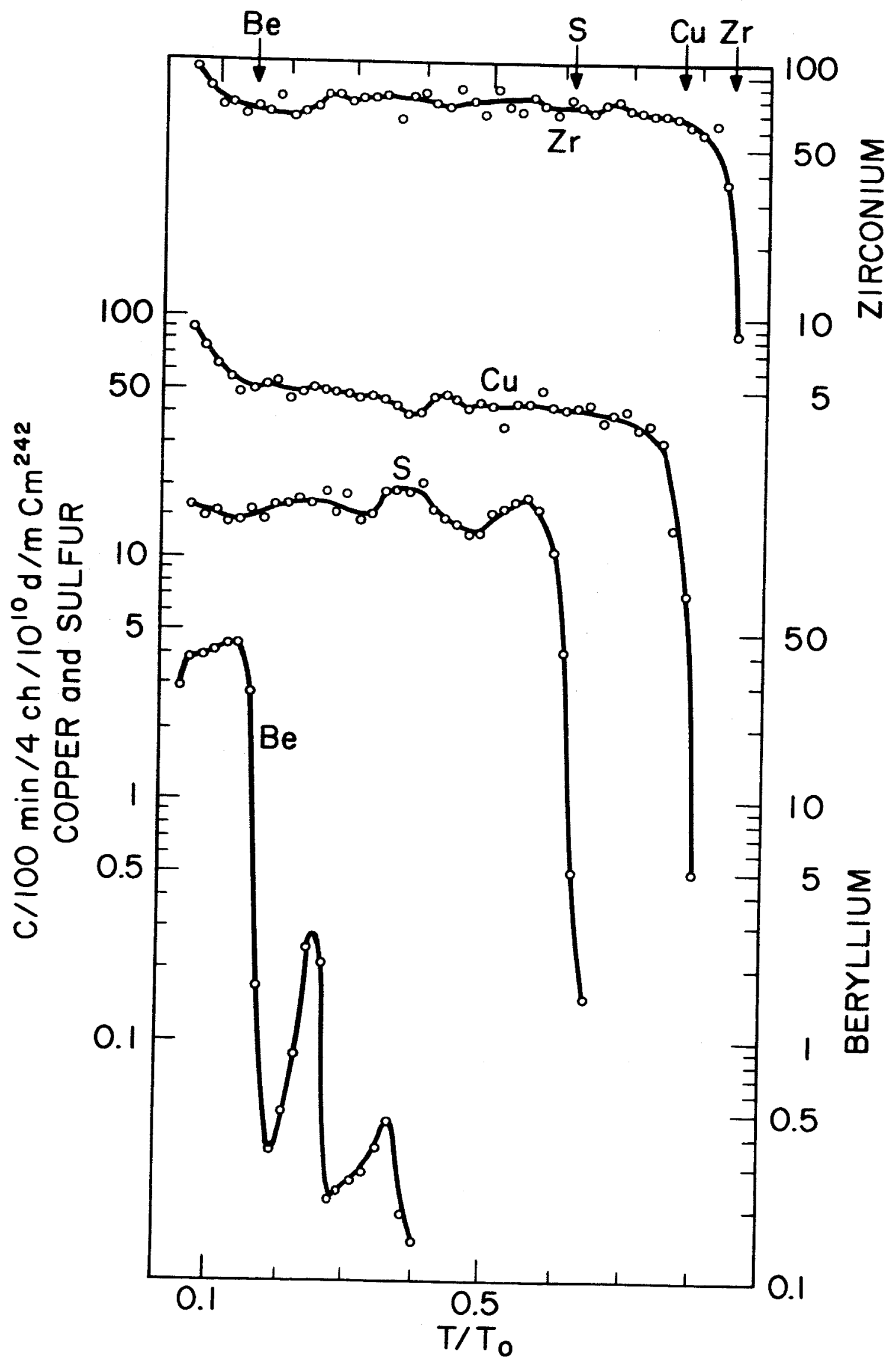




\section{FIGURE 4}

Alpha scattering spectrums from some oxides measured with the Research Apparatus. See Figure 3 for comments on the abscissas and ordinates. Data are presented for $\mathrm{Al}_{2} \mathrm{O}_{3}, \mathrm{SiO}_{2}, \mathrm{TiO}_{2}$ and $\mathrm{Fe}_{2} \mathrm{O}_{3} \cdot$ The positions of the expected high energy endpoints according to Equation 1 are indicated by arrows. 


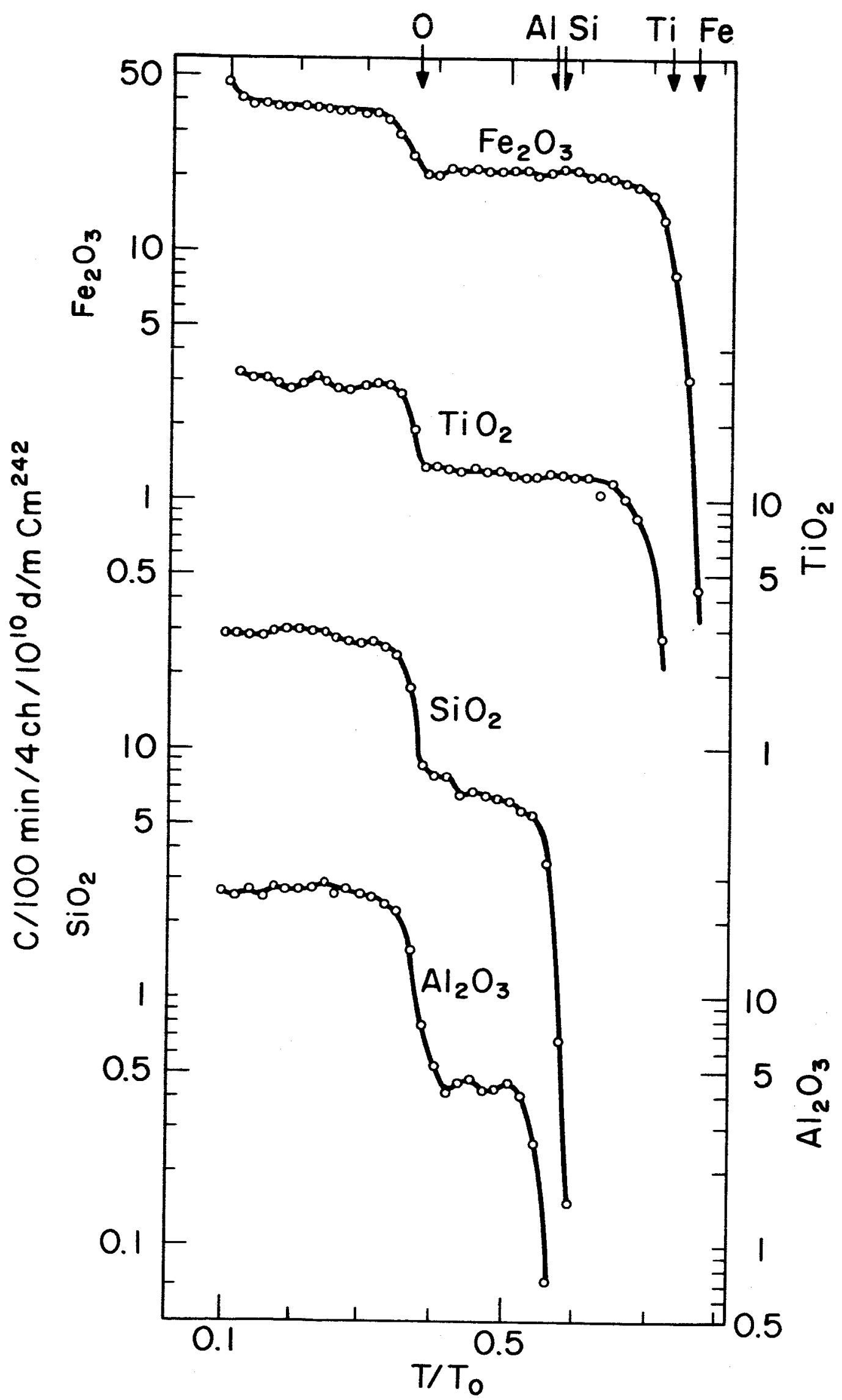


scattered from a series of oxides $\left(\mathrm{Al}_{2} \mathrm{O}_{3}, \mathrm{SiO}_{2}, \mathrm{TiO}_{2}\right.$ and $\left.\mathrm{Fe}_{2} \mathrm{O}_{3}\right)$. Fig. 5 shows comparable spectra from a selection of other slightly more complex compounds -- the sodium salts of some oxyacids $\left(\mathrm{Na}_{2} \mathrm{CO}_{3}, \mathrm{Na}_{3} \mathrm{PO}_{4}, \mathrm{Na}_{2} \mathrm{SO}_{4}, \mathrm{Na}_{2} \mathrm{CO} \mathrm{Q}_{4}\right)$.

The spectra from the oxides (Fig. 4) are characterized by two regions; in the low energy region the scattering is due to oxygen and the metal. The oxygen contribution stops at around $\mathrm{T} / \mathrm{T}_{\mathrm{o}}=0.37$, and the region beyond this is determined entirely by the metal with its characteristic endpoint. Similarly in Fig. 5 and spectra for more complicated systems, the spectra appear to be made up of additive contributions from the contributing elements, each with its own characteristic spectrum and endpoint, although limitations of intensity and resolution often preclude easy visual identification. Figs. 6 and 7 presents data on some complex systems, black dirt, clay - Fig. 6 - and olivene, a chondritic meteorite (Bruderheim) and a carbonaceous chondrite (Mighei) - Fig. 7 .

The intensities of protons from $\alpha, p$ reactions are usually much lower than thot of the scattered alpha particles. However, only a small number of elements have an appreciable yield of $(\alpha, p)$ reactions with 6.11 Mev alpha particles and these proton spectra have very characteristic shapes. A few proton spectra from $(\alpha, p)$ reactions on thick targets, obtained with the prototype instrument, are shown in Fig. 8. Other experiments with the 


\section{FIGURE 5}

Alpha scattering Spectrums from the sodium salts of some oxyacids obtained with the Research Apparatus. See Figure 3 for comments on the ordinates and abscissas. Data are presented for $\mathrm{Na}_{2} \mathrm{CO}_{3}, \mathrm{Na}_{3} \mathrm{PO}_{4}, \mathrm{Na}_{2} \mathrm{SO}_{4}, \mathrm{Na}_{2} \mathrm{CrO}_{4}$. The positions of the expected high energy endpoints according to Equation 1 are indicated by arrows. 


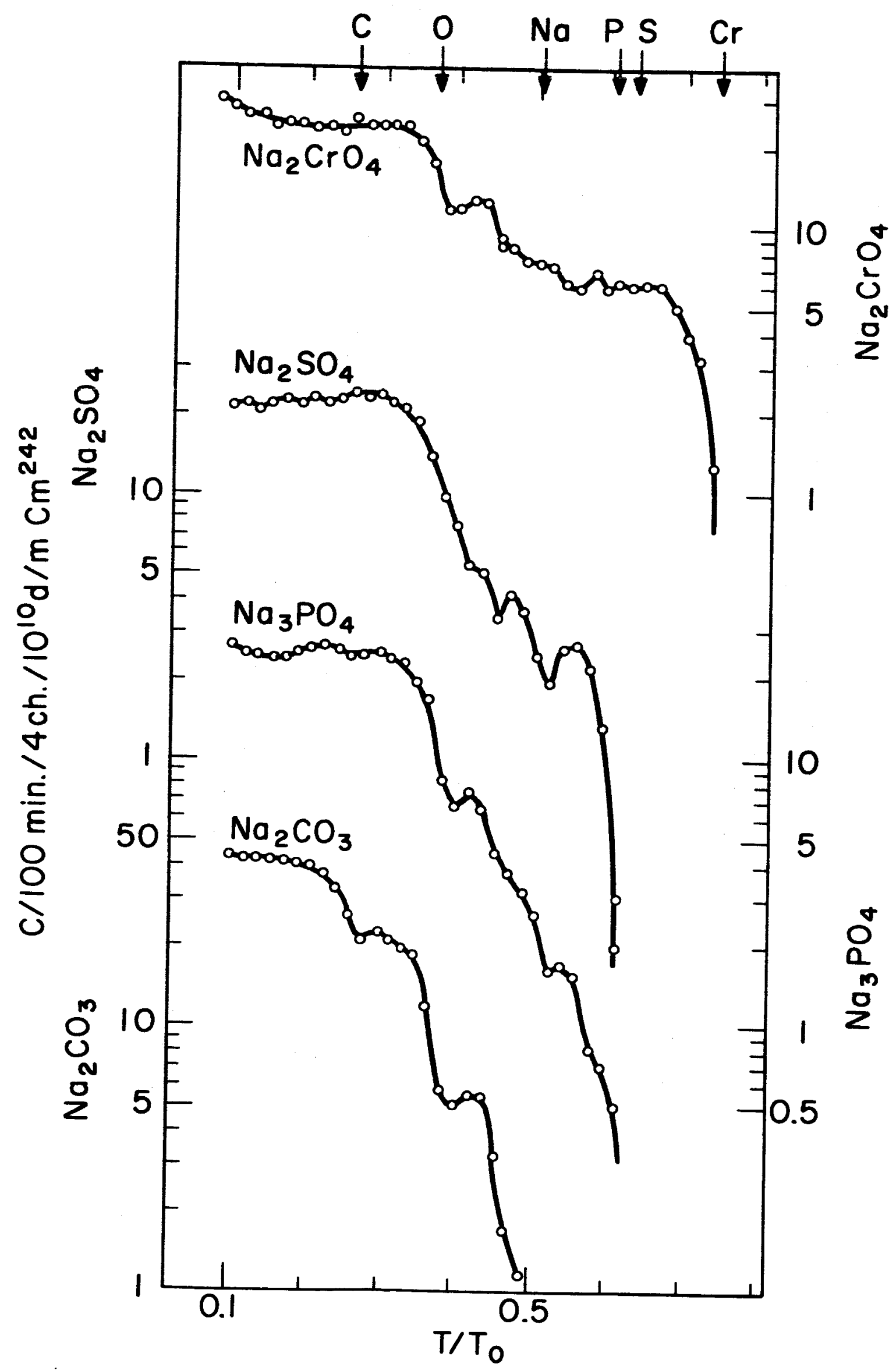




\title{
FIGURE 6
}

\begin{abstract}
Alpha scattering spectrums from terrestrial earths obtained with the Research Apparatus. Data are presented for black dirt and clay obtained at the Argonne National Laboratory site. See Figure 3 for comments on the ordinates and abscissas. The more prominent features of the scattering spectrums may be tentatively assigned to the presence of elements the positions of whose high energy endpoints (Equation 1) are indicated by arrows.
\end{abstract}




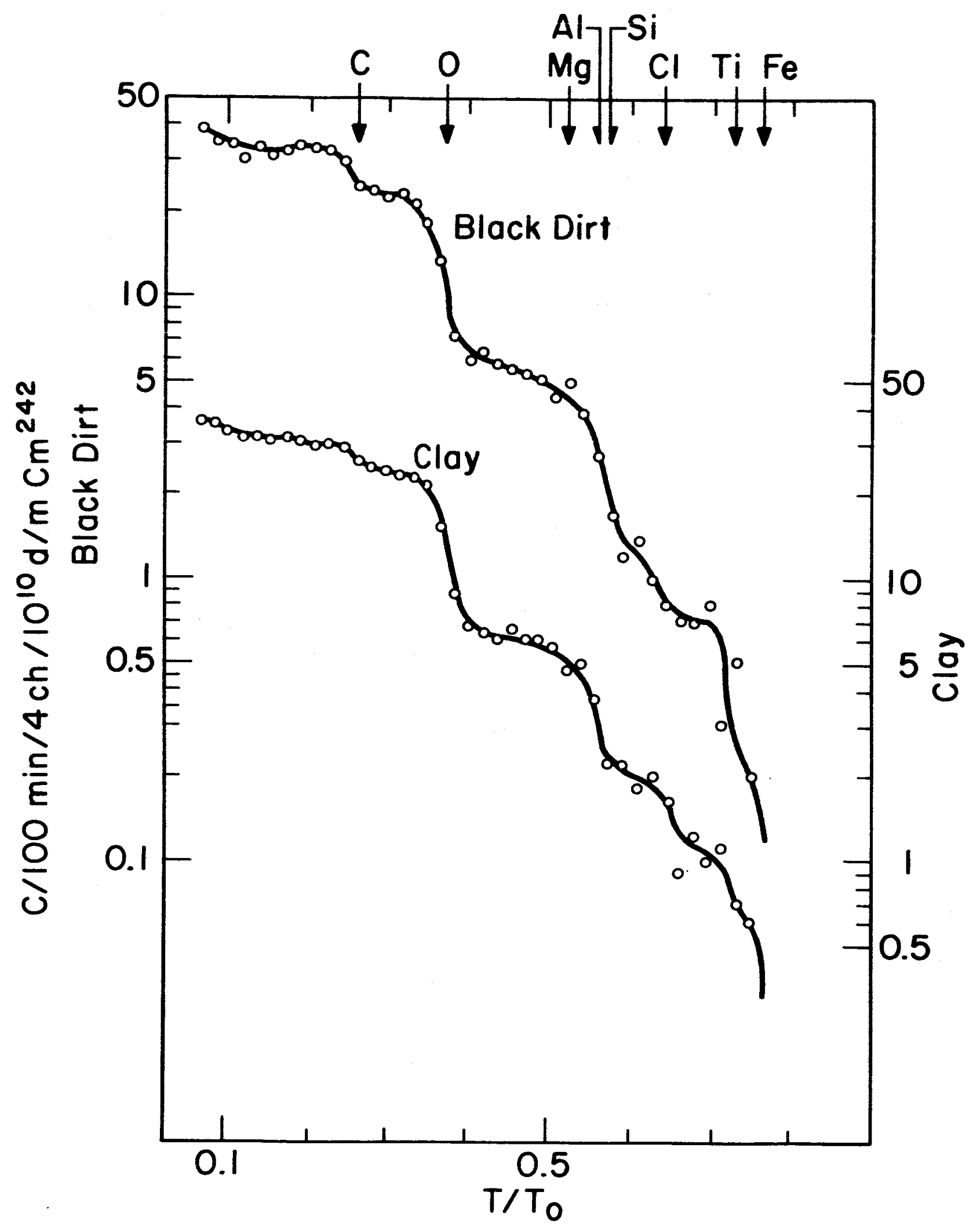




\section{FIGURE 7}

Alpha scattering spectrums from some materials of geochemical interest obtained with the Research Apparatus. The abscissas are channel numbers (Iinearly related to the energy of the scattered alpha particles). The ordinates are intensities on an arbitrary scale. Data are presented for:
A. Olivene
B. Bruderheim (a chondritic meteorite)
C. Mighei (a carbonaceous chondrite)

The arrows indicate the positions of the theoretical high energy endpoints (Equation 1) for some elements that are expected to be present. 


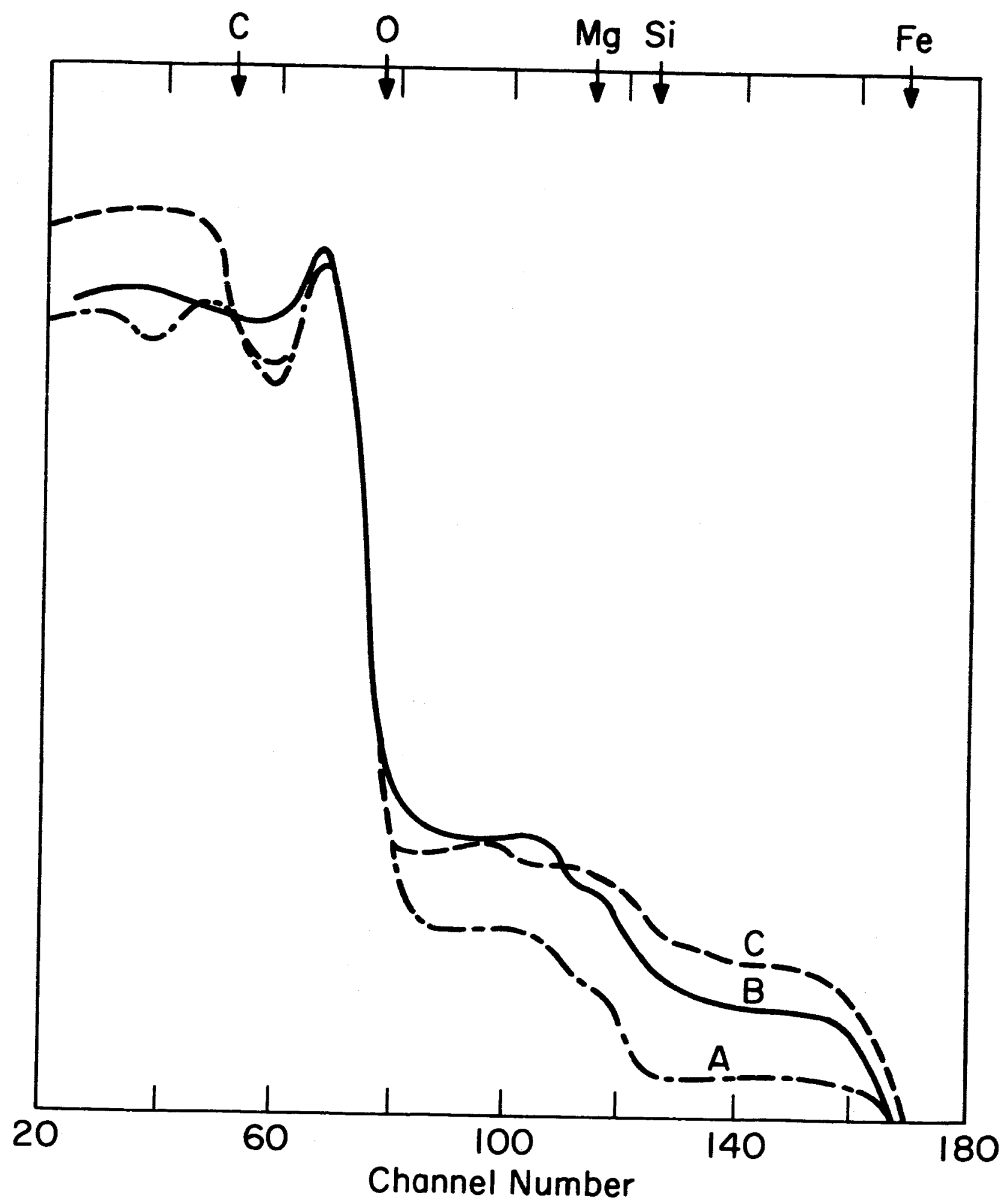




\section{FIGURE 8}

Protons from $(\boldsymbol{\alpha}, \mathrm{p})$ reactions from various elements. These data were obtained with the Prototype Instrument using a source strength of approximately $1.4 \times 10^{9} \mathrm{~d} / \mathrm{m}$ of $\mathrm{Cm}^{242}$. The detectors were covered with gold foils (approximately $0.6 \mathrm{mil}$ thick) to remove the scattered alpha particles. The ordinates are counts per 100 min per 4 channels of the analyzer; the abscissas are channel numbers -- linearly related to the energy of the protons. The background in the system has already been subtracted. Data are present for:
A. Carbon in the form of graphite
B. Nitrogen in the form of $\mathrm{NH}_{4} \mathrm{NO}_{3}$
C. Fluorine in the form of tefion
D. Sodium in the form of $\mathrm{Na}_{2} \mathrm{CO}_{3}$
E. Magnesium metal
F. Aluminum metal 


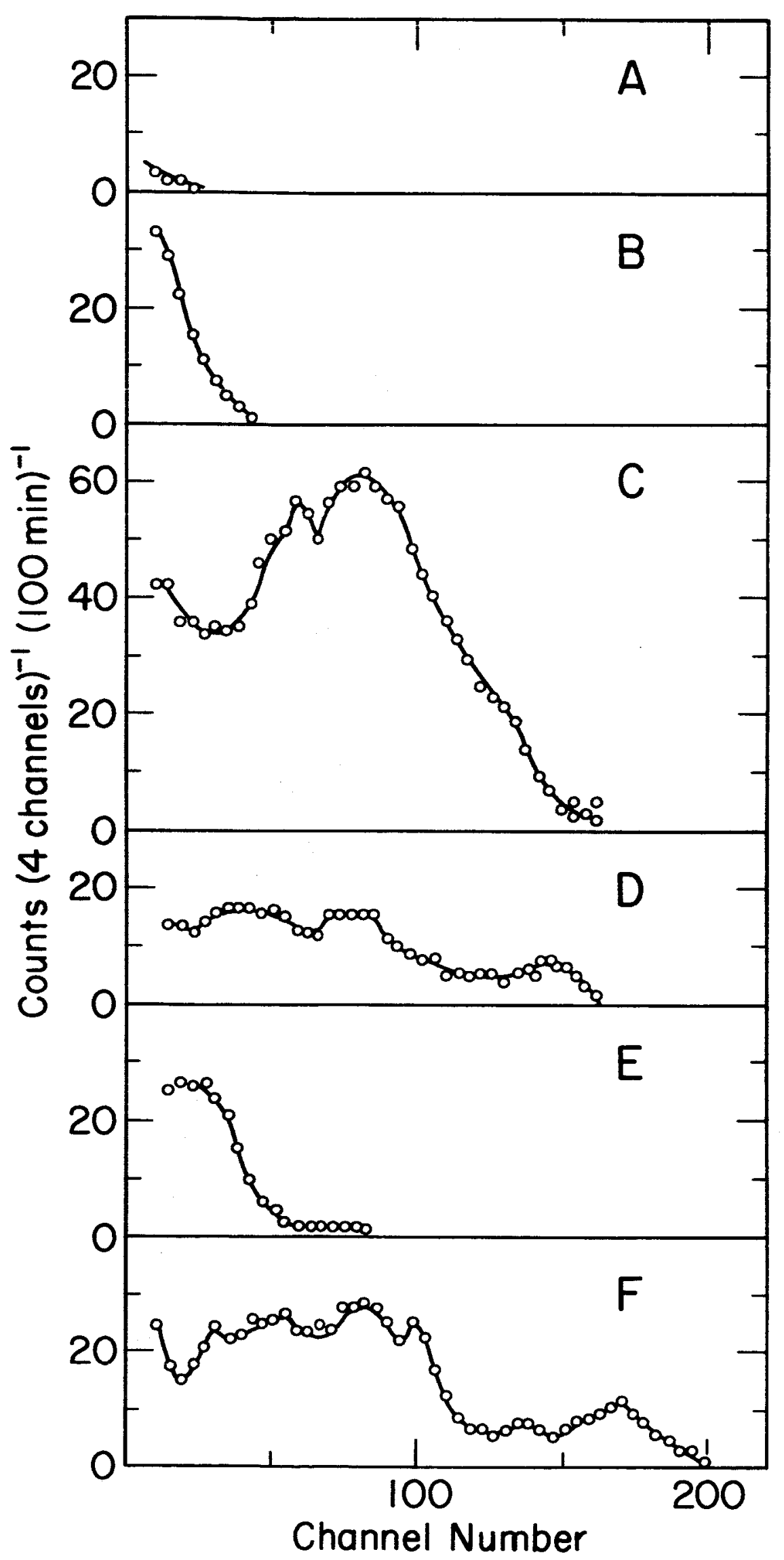


research and breadboard instruments confirm the general yield pattern as well as structure shown by these curves. They also confirm the expectation that there should be no protons emitted by carbon, oxygen, or heavy elements such as iron. A good yield of protons is also obtained from boron, and a moderate yield from silicon fwith about the same energy as those from magnesium).

\section{DISCUSSION}

\section{A. Alpha Scattering Spectra}

It is evident from Figure 3 that the alpha scattering spectra from pure elements fall into two categories. For high $\mathrm{Z}$ (greater than about 20 ) the spectra are nearly rectangular in shape with a sharp, high energy cutoff.' This high energy cutoff is given, from conservation of energy and momentum, by the equation (Rutherford, Chadwich and Ellis, 1930)

$$
f(A, \theta)=\frac{T_{\max }}{T_{0}}=\frac{\left(\frac{4 \cos \theta}{A}+\sqrt{1-\frac{16}{A^{2}} \sin ^{2} \theta}\right)^{2}}{(1+4 / A)^{2}}
$$

where $\theta$ is the angle of scattering in the laboratory system ( $160^{\circ}$ in our experiments) and $A$ is the mass number of the scatterer. The numerical values at $160^{\circ}$ are not much different from those at $180^{\circ}$ where the formula reduces to

$$
f\left(A, 180^{\circ}\right)=\frac{T_{\max }}{T_{9}}=\left(\frac{A-4}{A+4}\right)^{2}
$$


A plot of Equation 1, for various interesting values of A, is shown as a function of the laboratory scattering angle in Figure 9. According to these formulas the high energy endpoints are sensitive functions of the mass number for low A.

The alpha scattering spectra from elements of low $\mathrm{Z}$ (e.g. C, Mg, Al) show structure (to be discussed below) but the high energy endpoint still exists, although in some cases it is harder to establish. Figure 10 illustrates the agreement between the theoretical and experimental high energy endpoints. The observed energy of the endpoints, as determined by the channel number of the multichannel analyzer, is plotted versus $f(A, \theta)$ as given by Equation 1 . It is seen that the experimental values lie very close to a straight line connecting zero energy and the original energy of the alpha particles (plotted at $\mathrm{T}$

$\left.\frac{T_{\max }}{\mathrm{T}_{(}}=1\right)$. The figure also illustrates the good dispersion between the endpoints of neighboring light elements (C, N, O, F). The separation between the endpoints of adjacent elements decreases as A increases. Most of the instruments used to date have had overall resolutions of about 3 percent. This means that for atomic weights greater than about 25, individual isotopes are hard to resolve. Elemental analysis is further complicated by the presence of several isotopes in the heavier elements and by the presence of several isotopes of the same mass number in different elements. In practice, the usefulness 


\section{FIGURE 9}

Dependence of the maximum energy of scattered alpha particles, $T_{\max } / T_{0}$, on the laboratory angle of scattering, $\theta$, and on the mass number, A. See Equation 1 of text. 


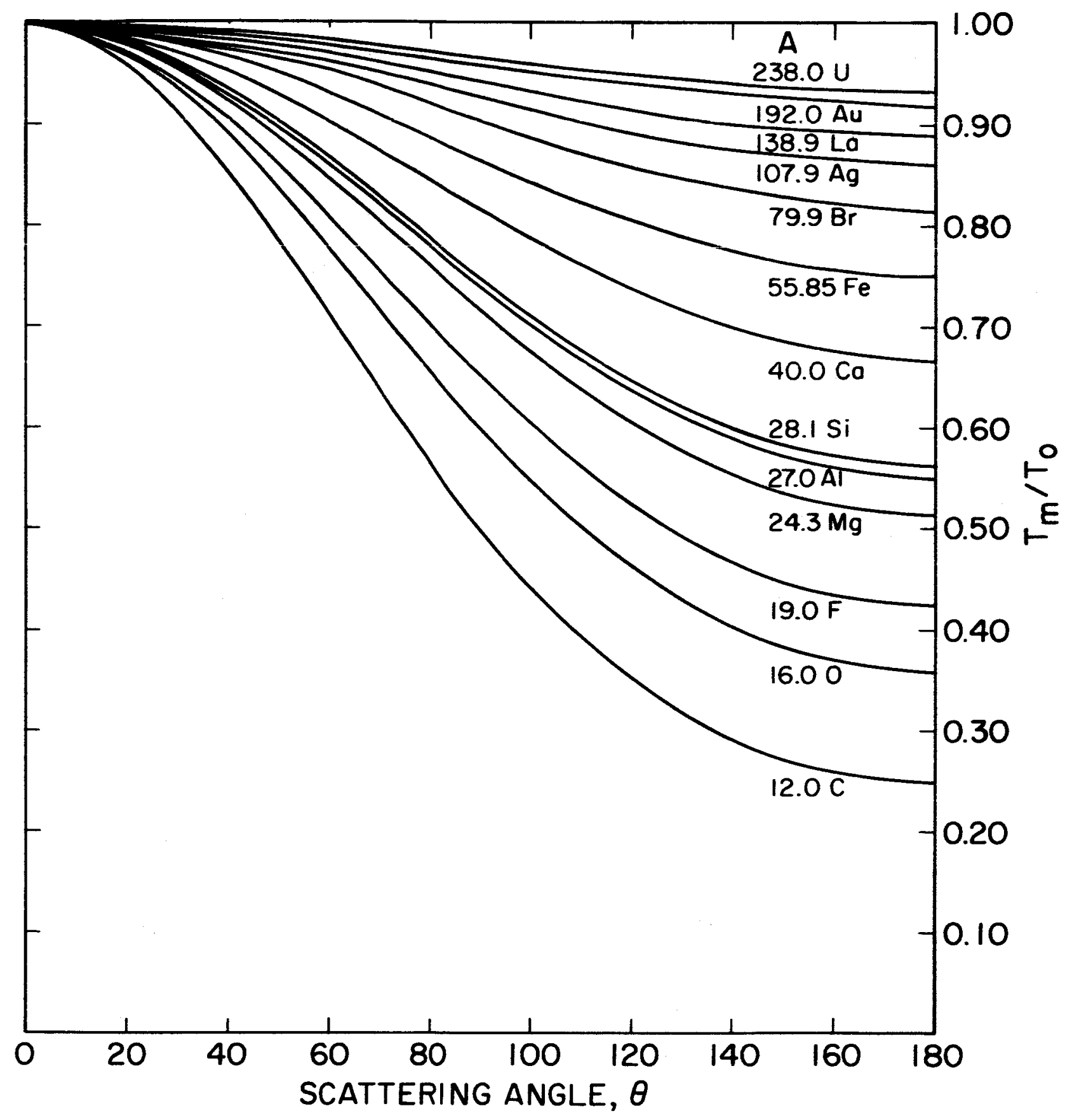




\section{FIGURE 10}

Agreement of observed and theoretical endpoints of the scattering spectrums of various elements as obtained with the Research Apparatus. The straight line, drawn through $\mathrm{T}_{\max } / \mathrm{T}_{\mathrm{O}}=1$ at channel 224 (the channel of the peak energy of the incident alpha particles) indicates how well the experimental endpoints agree with the predictions of Equation 1. The negative intercept at $\mathrm{T}_{\max } / \mathrm{T}_{0}=0$ is consistent with the positive energy threshold of the analyzer. 


\section{FIGURE 4}

Alpha scattering spectrums from some oxides measured with the Research Apparatus. See Figure 3 for comments on the abscissas and ordinates. Data are presented for $\mathrm{Al}_{2} \mathrm{O}_{3}, \mathrm{SiO}_{2}, \mathrm{TiO}_{2}$ and $\mathrm{Fe}_{2} \mathrm{O}_{3}$. The positions of the expected high energy endpoints according to Equation 1 are indicated by arrows. 


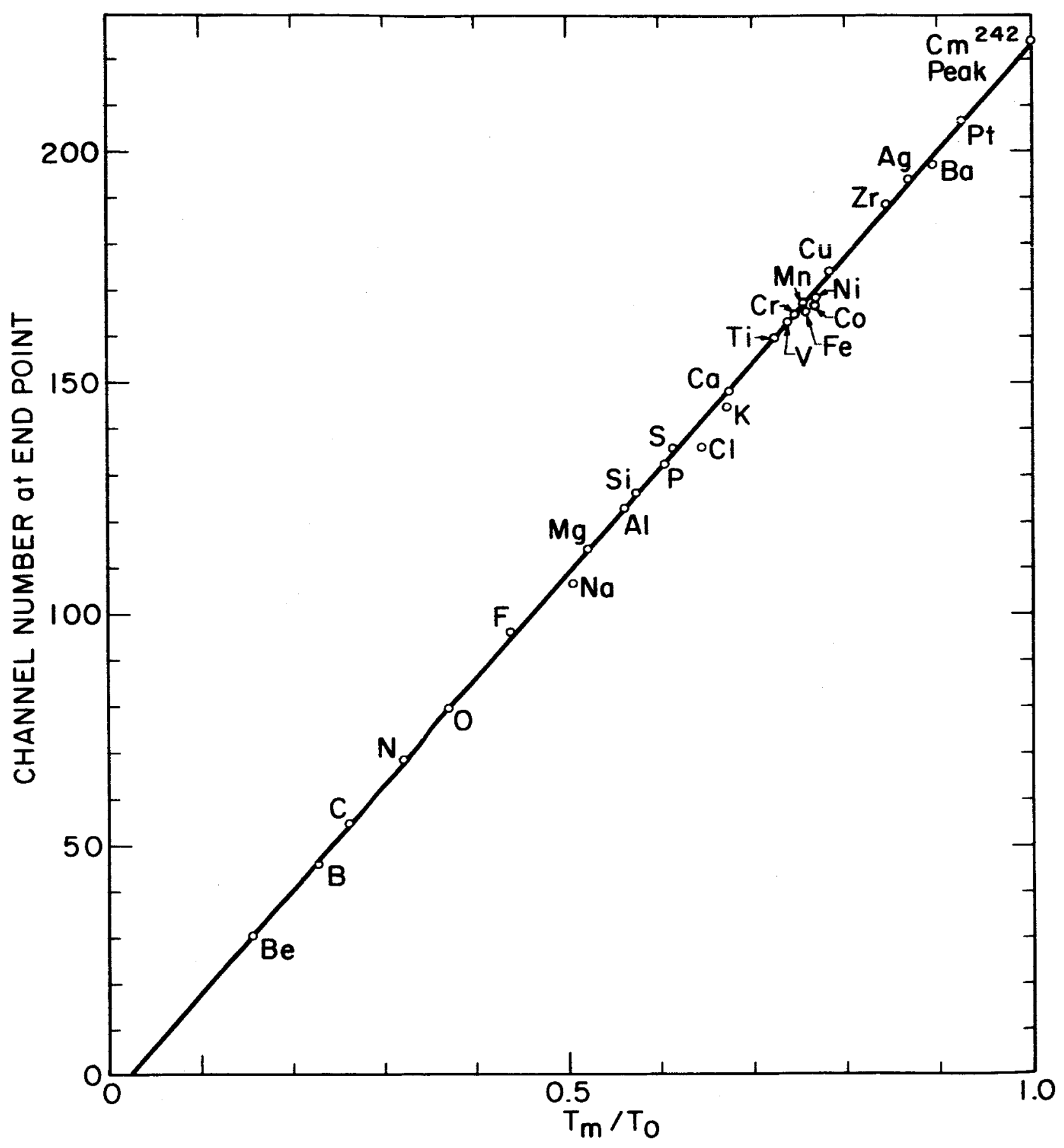


of the technique in distinguishing neighboring elements is not degraded as rapidly as might be expected because of the usually low abundances of some elements such as argon and scandium, and because the sensitivity for heavier elements increases with $\mathrm{Z}$ (see below).

For the case of elements of high $Z$, the shape of the spectra and the intensities are determined by the Rutherford scattering of the alpha particles. In a particular geometry this is, in principle, completely calculable from theoretical coulomb scattering cross sections and the energy loss characteristics of the substance being investigated. For thick targets of the type studied here a detailed calculation of this type is complicated by the incompletely known energy loss cross sections, particularly at low energies, and by the occurrence of multiple scattering - also most important at low energies.

For the purposes of this paper the theoretical interpretation of alpha particle scattering from a thick target will be done in terms of the diagram of Figure 11. This interpretation will assume only single scattering contributions. In Figure 11, $\mathrm{T}_{\mathrm{O}}$ is the energy of a parallel alpha particle beam incident perpendicularly, on a homogeneous thick target. (The case of noniperpendicular incidence can be readily shown to be not significantly different.) In this 


\section{FIGURE 11}

Geometrical relationships in alpha particle scattering from a thick target. In this diagram the distances from the source and detector to the sample are assumed to be very large (centimeters) compared to the penetration of the alpha particles into the sample (microns). 


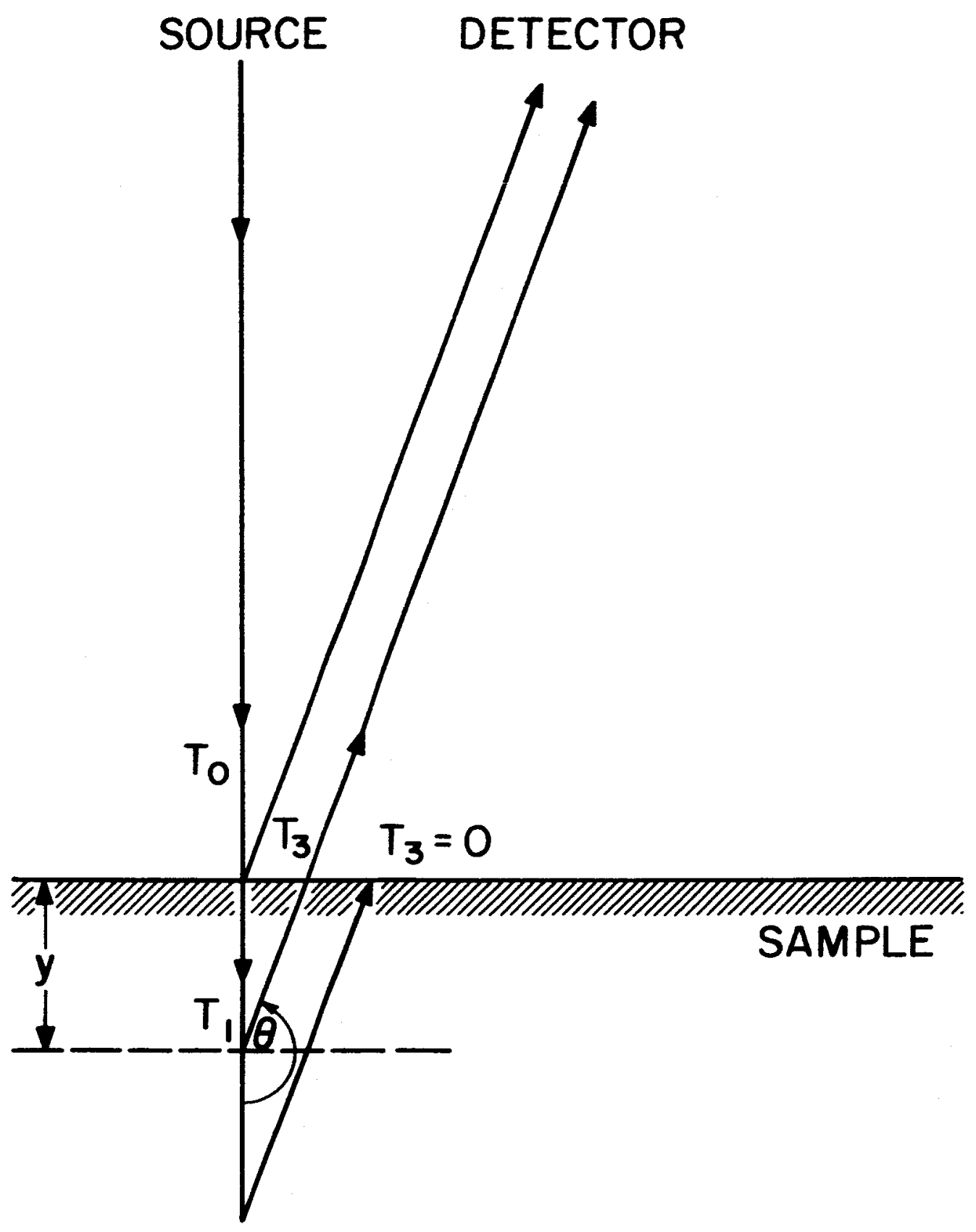


diagram, $\mathrm{T}_{3}$ is the energy with which the alpha particles emerge after having been scattered through an angle $\theta$. In order to emerge with an energy $\mathrm{T}_{3}$, the alpha particles must have been scattered at a depth $\mathrm{y}$, which is related to $\mathrm{T}_{0}, \mathrm{~T}_{3}$ and $\theta$ through the stopping characteristics of the medium, and the energy change upon scattering, $f$ (Equation 1 ), via the relation:

$$
y=\int_{T_{0}}^{T}\left(\frac{\frac{1}{d T}}{d x}\right) d T=-\cos \theta \int_{f T_{1}}^{T_{3}} \frac{1}{\left(\frac{d T}{d x}\right)} d T
$$

where $\left(\frac{d T}{d x}\right)$ is the energy change due to ionization in the material (e.g. in Mev $\mathrm{cm}^{-1}$ ). These relations determine $\mathrm{T}_{1}$ and $\mathrm{y}$, for a given $\theta, \mathrm{T}_{0}, \mathrm{~T}_{3}$, and scattering substance.

The scattered spectrum at an angle $\theta$ is the number of alpha particles as a function of energy, $\mathrm{T}_{3}$. It is given, theoretically, by the expression

$$
\frac{d I}{d T_{3}}=I_{0} n \sigma\left(T_{1}\right) \frac{d y}{d T_{3}} \cdot
$$

In this expression

$$
\begin{aligned}
\frac{\mathrm{dI}}{\mathrm{dT}_{3}=} & \text { intensity of scattered particles per steradian } \\
& \text { at an angle } \theta \text { and per unit emergent energy, } \mathrm{T}_{3} \\
\mathrm{I}_{0}= & \text { intensity of incident particles } \\
\mathrm{n}= & \text { number of scattering nuclei } \mathrm{x} \mathrm{cm}^{-3}
\end{aligned}
$$


$\sigma(T)=$ cross section for scattering alpha particles by the nuclei of interest at energy $T$, (in $\mathrm{cm}^{2}$ nuclei-1 sterad $^{-1}$ ). For heavy elements, this cross section is the Rutherford scattering cross section: $\sigma\left(\mathrm{T}_{1}\right)=\frac{\mathrm{z}^{2} \times 5.184 \times 10^{-27}}{\mathrm{~T}_{1}{ }^{2}(\mathrm{MeV})} \frac{1}{\sin ^{4} \theta / 2}$ where $\mathrm{Z}$ is the atomic number of the scattering nucleus. (For heavy elements the difference between laboratory and center of mass scattering angles is negligible).

$\frac{\mathrm{dy}}{\mathrm{dT}_{3}}=$ variation in depth (at which the scattering occurs) with change in emergent energy.

$\frac{d y}{d T_{3}}=\frac{1}{\left(\frac{d T}{d x}\right)_{T_{3}}\left[\frac{1}{-\cos \theta}+\frac{\mathrm{f}\left(\frac{d T}{d x}\right) T_{1}}{\left(\frac{d T}{d x}\right) \mathrm{fT}_{1}}\right]}$

$\mathrm{T}_{1}=$ energy just before scattering

$\mathrm{fT}_{1}=$ energy just after scattering

Thus, the intensity of scattering can be written as

$$
\frac{d I}{d T_{3}}=\frac{I_{0} n z^{2} \times 5.184 \times 10^{-27}}{T_{1}^{2}(M e v) \sin ^{4} \frac{\theta}{2}} \frac{1}{\left(\frac{d T}{d x}\right)} \frac{1}{T_{3}} \frac{1}{\left[\frac{f\left(\frac{d T}{d x}\right) T_{1}}{-\cos \theta}+\frac{\left(\frac{d T}{d x}\right) T_{1}}{1}\right]}
$$


For alpha particles of energy above a few Mev, Equation 4

$$
\frac{\mathrm{dT}}{\mathrm{dx}}=-\frac{\mathrm{k}}{\sqrt{\mathrm{T}}}
$$

is a frequently used approximation to the energy loss due to ionization (Friedlander \& Kennedy, 1949). Use of such an approximate energy loss formula and the energy dependence of the coulomb scattering cross section, leads to a thick target scattering curve at $180^{\circ}$ illustrated in Figure $12 \mathrm{~b}$ by curve $\mathrm{C}$. The shape of this spectrum is independent of the target material except in the position of the high energy endpoint. For example, the spectrum would end at a $\mathrm{T} / \mathrm{T}_{0}$ value of $0.77^{8}$ for scattering from copper, and at 0.55 for aluminum.

Literature data on energy loss of alpha particles at low energies are not always consistent, and in any case are sparse for alpha particles of energy less than $2 \mathrm{Mev}$ (see, e.g., Whaling, 1958). A more realistic energy loss function than one varying inversely as the square root of the energy (curve $A$ of Figure 12a) is illustrated by the curve B of Figure 12a, which shows the energy loss going through a maximum at a little over $0.5 \mathrm{Mev}$. Use of this function, together with Equation 3, leads to a calculated scattered spectrum shown as curve $D$ of Figure 12b. This curve was calculated for copper as a target. It is seen that a more realistic energy loss relation may lead 


\section{FIGURE 12}

Relationship of Energy Loss Function to Spectrum of Backscattered Alpha Particles.

a. Energy loss of alpha particles (arbitrary unitś) as a function of energy, in Mev. Curve A represents the function $\frac{d T}{d x}=-\frac{k}{\sqrt{T}}$; Curve $B$ represents an energy loss function for copper constructed from experimental data (Whaling, 1958).

b. Theoretical spectra of backscattered alpha particles calculated from Equation 3 for an angle of $180^{\circ}$. The ordinates are arbitrary units; the abscissas are energies of alpha particles in units of fractions of the original energy, $T / T_{0}$. Curve $C$ assumes an energy loss corresponding to Curve A (above). Different elements would have the spectrum ending at $\mathrm{T} / \mathrm{T}_{\mathrm{O}}$ values indicated by arrows on the top scale. Curve $D$ is the calculated spectrum for copper using Curve $B$ (above) for the energy loss function.

c. Experimental scattering spectrum from iron obtained at an angle of $174 \pm 3^{\circ}$ in a special apparatus. The ordinates are arbitrary intensity units; the abscissas are the same as in $b$ (above). 

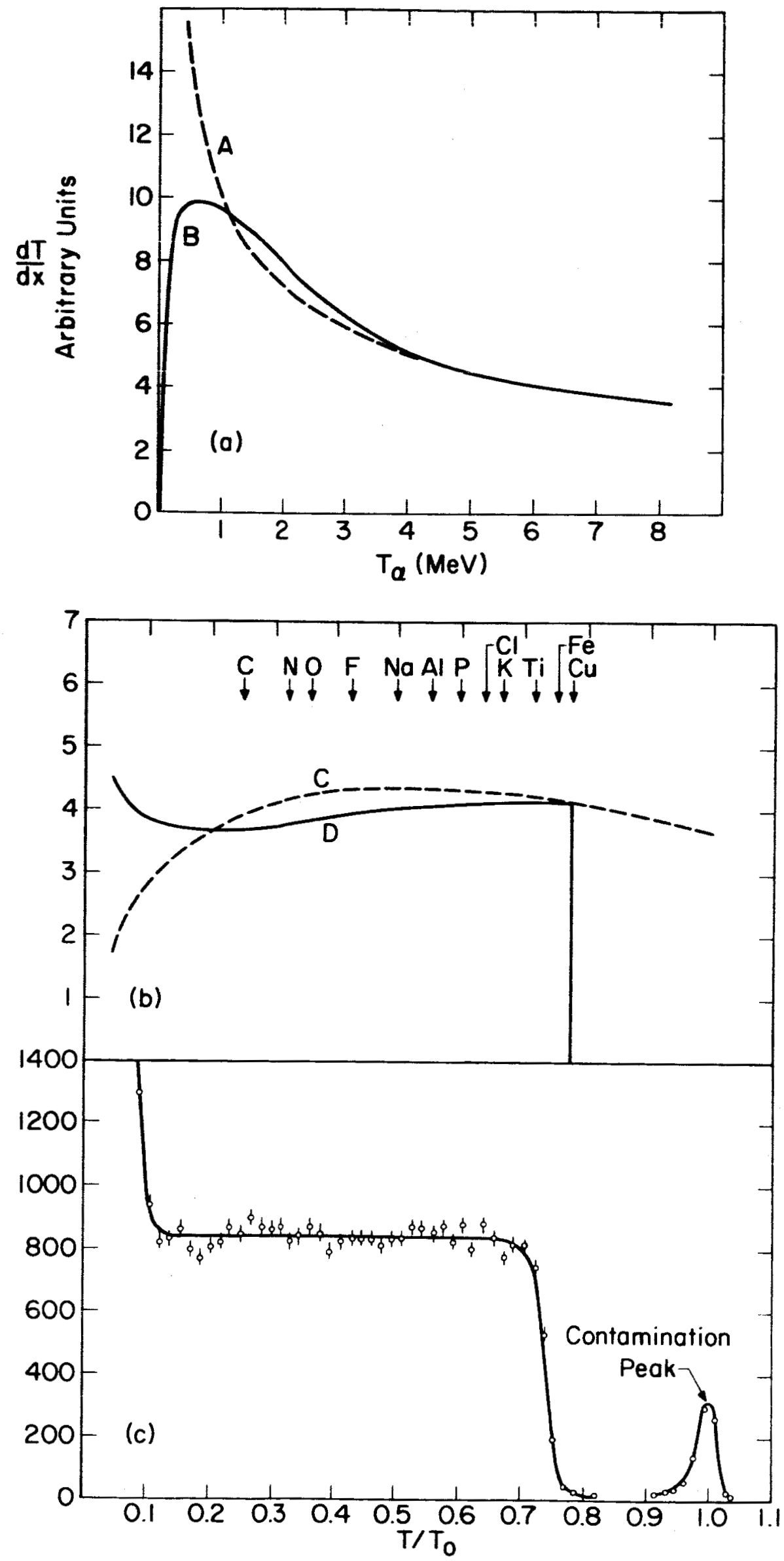
to an actual rise in the scattered spectrum at very low energies.

For comparison with these theoretical curves, Figure 12c shows the spectrum of alpha particles scattered from iron at an average angle of $174 \pm 3^{\circ}$ obtained in a special experimental arrangement. The sharp high energy endpoint at the correct value of $\mathrm{T} / \mathrm{T}_{0}=0.75$ is evident as well as the essentially rectangular shape of the spectrum down to $\mathrm{T} / \mathrm{T}_{0}$ values of about 0.1 . At present it is not clear whether the differences, in detail, between curve $D$ of Figure $12 b$ and the experimental curve are due to the inaccurately known energy loss function, or due to the contribution of multiple coulomb scattering at low energies.

In ppactical situations, such as obtain in the different instruments used in this work, there is a considerable variation in scattering angles and paths. The calculation could still be performed, but would be laborious, and has not been done as yet. However, the empirically obtained scattering curves from elements heavier than silicon (see Figure 3) show an essentially rectangular shape similar to that calculated for better defined geometries. As already noted they have the correct high energy endpoints. We conclude that the observed shapes of the scattered spectra for high $\mathrm{Z}$ elements are consistent with Rutherford scattering cross sections and stopping powers of materials to the extent that they are known. 
The intensities of Rutherford scattering from different elements, can best be compared near the high energy endpoints of the respective scattered spectra. In this energy region, the energy loss is approximately representable by Equation 4, with the constant, $k$, being roughly related to the atomic number of the material by the relation (Whaling, 1958)

$$
\bar{k}=n k_{0} \sqrt{z}
$$

The scattered spectrum near the high energy endpoint represents scattering from the surface of the material, and so $\mathrm{T}_{1}=\mathrm{T}_{0}$ and $\mathrm{T}_{3}=\mathrm{fT}_{0}$. Equation 3 then simplifies to:

$$
\left(\frac{d I}{d T_{3}}\right)_{T_{3}}=f T_{0}=\frac{I_{0} \operatorname{const}(\theta)}{T_{0}^{3 / 2}} z^{3 / 2} \frac{\sqrt{f}}{k\left(1-f^{3 / 2} \cos \theta\right)}
$$

where

$$
\begin{aligned}
\left.\left(\frac{\mathrm{dI}}{\mathrm{dT}}\right)_{3}\right)_{3}=\mathrm{fT}_{0}= & \text { intensity of scattered alpha } \\
& \text { particles near the high energy } \\
& \text { endpoint. }
\end{aligned}
$$

For negative values of $\cos \theta$ (applicable for backscattering) and values of A larger than about 20, formula 6 becomes nearly independent of $\mathrm{A}$, and predicts an intensity of scattering, near the endpoints, which varies as $\mathrm{z}^{3 / 2}$. Figure 13 presents experimental data on this relationship. In this figure, the logarithm of the intensity of scattering near the high energy endpoint is plotted as a function of the logarithm of the 


\section{FIGURE 13}

Dependence of intensity of scattering on atomic number. The ordinates are intensities (on a logarithmic scale) of scattering near the endpoint by different elements. The units are the same as those of Figure 3. The abscissas are atomic numbers (on a logarithmic scale). In the case of elements not readily available in suitable elementary form (e.g. Na, Cl) the intensities were obtained by the procedure described in section $\mathrm{C}$ of the discussion. Points obtained in this way are indicated by open circles. In some cases several compounds were used. The straight line has been drawn through the high $z$ points with a slope of $3 / 2$. 


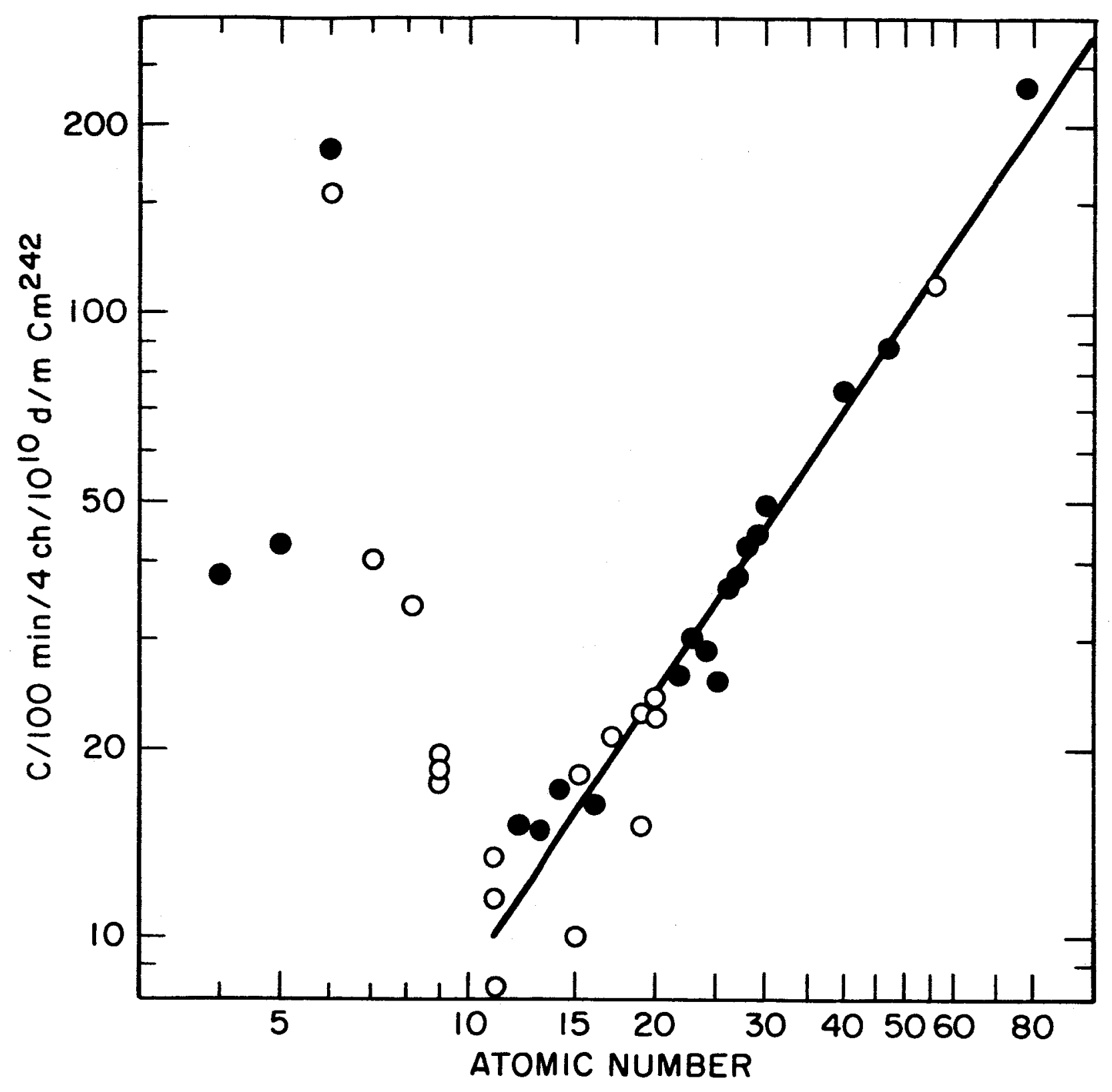


atomic number. Above a $\mathrm{z}$ of $1 \mathrm{I}$ the points cluster about a line of slope $3 / 2$ indicating satisfactory agreement with the predictions of the theory. As the $z$ of the element is decreased below that of sodium, the regular behavior disappears, the intensity of scattering becoming much higher than that predicted from Rutherford scattering, and varying irregularly from element to element. It is especially high for carbon.

The departure from the $z^{3 / 2}$ law for the intensity of scattering at low $Z$ is associated with an increase in structure of the scattering curve as a function of energy (see Figure 3). Both features - the increased intensity and structure - are associated with specifically nuclear (as opposed to electrostatic) interactions of the incoming alpha particles with the target nuclei. These become dominant when the charge of the target nucleus becomes low enough for significant coulomb barrier penetration by the $6.11 \mathrm{Mev}$ alpha particles. As the alpha particle goes through the thick target it loses energy continuously and thus goes through energy regions where the nuclear cross section may become very large. At these resonant energies there will be enhanced scattering (or other nuclear effects). Examples of such resonances in nuclear scattering are well known. For example, Bittner and Moffat (1954) observed resonant scattering from $\mathrm{C}^{12}$ with $5.85 \mathrm{Mev}$ alpha particles. The scattering was about 15 times as large as Rutherford scattering at a laboratory angle of $167^{\circ}$ and was 
very angular dependent; it was many times lower at a scattering angle of $134^{\circ}$. Similarly, oxygen (Ferguson \& Walker, 1940) has resonant scattering for $5.5 \mathrm{Mev}$ alpha particles that is more than ten times greater at $157^{\circ}$ than Rutherford scattering.

We have investigated the angular dependence of the thick target scattering from the three elements present in calcium carbcnate. For this purpose the Research Apparatus was modified slightly to measure scattering at $140^{\circ}$. Table 2 presents the intensities of scattering at mean scattering angles of $160^{\circ}$ and $140^{\circ}$ attributable to carbon, oxygen and calcium in the gross scattering spectrum of calcium carbonate. It is seen from Table 2 that the intensity of scattering attributable to calcium increases slightly as the angle is decreased, whereas that for carbon and oxygen decreases markedly.

In conclusion, the intensities of alpha scattering by different elements in instruments of the type described in this report appear to be consistent with fundamental nuclear physics information, and data of the type illustrated in Figure 13 may be used to estimate the relative sensitivity of the alpha scattering technique for various elements. 
TABLE 2. Effect of Angle of Scattering on Intensities Attributable to Carbon, Oxygen, and Calcium

(Intensities* measured in Modified Research Apparatus)

\begin{tabular}{ccc}
\hline & & Mean Angle of Scattering \\
\cline { 2 - 3 } Element & $160^{\circ}$ & $140^{\circ}$ \\
\hline $\mathrm{C}$ & 28 & 10 \\
$\mathrm{O}$ & 21 & 5.5 \\
$\mathrm{Ca}$ & 8.2 & 11 \\
\hline
\end{tabular}

* The intensities in this table are in arbitrary but comparable units. The values were determined by resolution of the gross $\mathrm{CaCO}_{3}$ scattering spectrum into components attributable to the three elements. 
B. Proton Spectra from $(\alpha, p)$ Reactions

The energy spectra and yields of protons from $(\alpha, p)$ reactions are similarly a reflection of the cross sections for such reactions with different nuclei at alpha energies up to $6.11 \mathrm{Mev}$. Energetically, such reactions are impossible for $\mathrm{Be}^{9}$, $\mathrm{c}^{12}, \mathrm{c}^{13}$ or for $\mathrm{o}^{16}, \mathrm{O}^{17}, \mathrm{o}^{18}$. For elements with nuclear charge above 20 , the coulomb barrier penetration probability is small enough to lower the cross sections to unimportant values. For the other light elements such as $\mathrm{B}, \mathrm{N}, \mathrm{F}, \mathrm{Na}, \mathrm{Mg}, \mathrm{Al}$, and $\mathrm{Si}$, protons are produced with energies and in groups consistent with known nuclear masses and energy levels, although only crude energy resolution is available in the kind of apparatus used in this work.

It should be noted that protons from $(\boldsymbol{\alpha}, \mathrm{p})$ reactions may complicate the appearance of the alpha scattering spectrum. Thus, for example, the peak at $\mathrm{T} / \mathrm{T}_{0}=0.37$ of the a scattering spectrum for boron (see Figure 3b) is at a higher energy than can be assigned to alpha particles from boron; it is reasonably attributed to protons from the exothermic $(\alpha, p)$ reaction on one of the boron isotopes. In interpreting such proton peaks it is important to remember the relatively long range of protons, which may exceed the sensitive depth of semiconductor detectors biased primarily for alpha particle detection. Another possible explanation for the peak in the boron spectrum 
is that it arises from scattering by a thin layer of oxygen. Such an explanation is inconsistent with the observation of the same peak in compounds containing boron. It appears to be a coincidence that the sensitive depth of the detectors used in this work is such that moderate energy protons deposit about the same energy in the detectors as does an alpha particle scattered by oxygen.

Although the intensity of protons from $(\boldsymbol{\alpha}, \mathrm{p})$ reactions is often much lower than that of the scattered alpha particles, incorporation of a detection system sensitive only to protons enhances the sensitivity of an analytical instrument for many possible applications. For example, in the case of rock analyses, the amounts of aluminum and sodium often have great geochemical interest. Their frequently low abundances in the presence of large amounts of silicon and magnesium make the scattered alpha particle spectrum an insensitive basis for their determination. The proton spectra from aluminum and sodium, however, extend to much higher energies than do those from silicon and magnesium and their characteristic shapes provide further basis for differentiation.

C. Interpretation of Complex Spectra

Qualitative inspection of the spectra of oxides and more complex compounds (Figures 4 and 5) indicates that they are superpositions of the scattering spectra of the constituent 
elements. It is of importance for analytical applications to understand to what extent this superposition is exact and what are the quantitative relations between the intensity of an element in a compound and in the pure state.

In the approximation that the energy loss of an alpha particle due to ionization in all substances is a universal function of the energy - depending on composition only via a multiplicative constant - it is easily shown theoretically that the alpha scattering curve from a compound is composed additively of the contributions of the individual elements. This approximation does not assume any specific form for the energy loss function; only that the energy dependence is independent of chemical composition. The constants entering into this superposition are related to the concentrations and the ionization energy loss cross sections per atom of the elements present. The proof of this follows:

From Equation 3, the ratio of intensity of scattering by an element, $i$, in a compound to that for the pure element, is

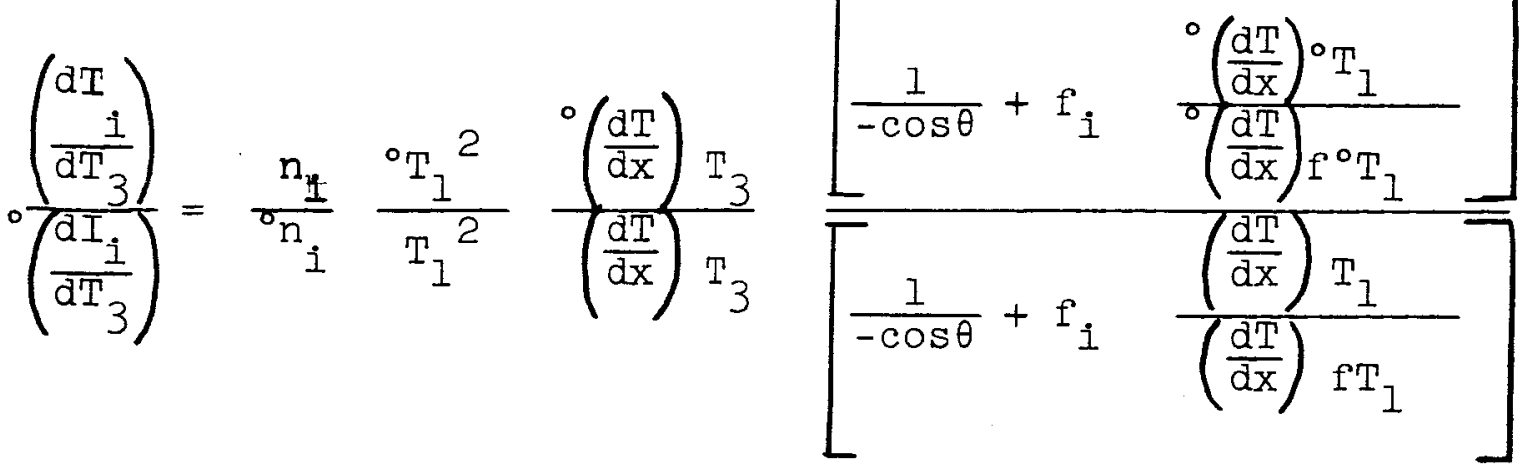


In this equation, the presuperscript ${ }^{\circ}$ denotes the value of a quantity in the pure element. It should be noted that the intensities are being compared at the same emergent energy $\mathrm{T}_{3}$, but that $\mathrm{T}_{1}$ and ${ }^{\circ} \mathrm{T}_{1}$ are not necessarily equal.

The assumption that the ionization energy loss is a universal function of the energy is equivalent to the equation:

$$
\left(\frac{d T}{d x}\right)_{T}=C_{i}{ }^{\circ}\left(\frac{d T}{d x}\right)_{T}
$$

where $C_{i}$ is a constant relating the energy loss per $\mathrm{cm}$ in the compound to that in the pure element. In the energy regions where this equation holds, ${ }^{\circ} \mathrm{T}_{1}=\mathrm{T}_{1}$, and the ratio of intensities then becomes

$$
\frac{\left(\frac{d I_{i}}{d T_{3}}\right)}{\left.\frac{d I_{i}}{d T_{3}}\right)}=\frac{n_{i}}{{ }^{0} n_{i}} \frac{1}{C_{i}}
$$

Equation 9 is independent of the energy $\mathrm{T}_{3}$ and thus predicts that the shape of the contribution of an element $i$ to a scattering spectrum from a compound is the same as the shape of the scattering spectrum of the pure element.

The ratio of intensities is the product of the ratio of the number of $i$ atoms per $\mathrm{cm}^{3}$ and the ratio of energy losses per cm for the two substances. Simple manipulation converts this to 


$$
\frac{\left(\frac{d_{i}}{d T_{3}}\right)}{\left(\frac{d I_{i}}{d T_{3}}\right)}=\frac{x_{i} \varphi_{i}}{\bar{\varphi}}
$$

where

$$
\begin{aligned}
x_{i}= & \text { fraction of atoms of type } i \text { in the compound } \\
\varphi_{i}= & \text { energy loss cross section per atom of the } \\
& \text { pure substance } i \text { (in Mev } \mathrm{cm}^{2} \text { atom }{ }^{-1} \text { ) } \\
\bar{\varphi}= & \text { average energy loss cross section per atom } \\
& \text { for the compound: }
\end{aligned}
$$

$$
\bar{\varphi}_{i}=\Sigma x_{i} \varphi_{i}
$$

Equation 10 is the basis of the quantitative interpretation of complex alpha scattering spectra in the energy regions where the basic assumption - Equation 8 - is satisfied. It requires a knowledge of the relative energy loss cross sections per atom which can be obtained from compilations such as whaling (1958). A simple relation that seems to be valid for the energy region of interest and for not too heavy elements, is that $\varphi_{i}$ varies as the square root of the atomic number (Equation 5). Introducing this into Equation 10 leads to:

$$
\frac{\left.\left(\frac{\mathrm{dI}}{\mathrm{dT}}\right)_{3}\right)}{\left(\frac{\mathrm{dI}}{\mathrm{dT}} \mathrm{T}_{3}\right)}=\frac{x_{i} \sqrt{\mathrm{Z}_{i}}}{<\sqrt{\mathrm{Z}_{i}}>}
$$


where the symbol $\left\langle\sqrt{z_{i}}>\right.$ means the average value of $\sqrt{z}$ for a particular compound.

Aside from the fundamental assumption that multiple scattering effects are unimportant, the above derivation depends firstly on the assumption of the same energy dependence of the ionization energy loss for all substances, and secondly on the relation between the relative ionization energy loss and the atomic number. Although the available data (Whaling, 1958) indicate systematic deviations from both of the latter approximations, it does not appear to be warranted to take these into account at the present stage of the development of the analytical technique.

Experimental checks of these theoretical considerations can be made using available data. The dependence of the shape of the spectrum on the chemical state of an element may be first examined. Quantitative comparison of the iron scattering spectrum beyond the oxygen endpoint $\left(\mathrm{T}_{\mathrm{T}} \mathrm{T}_{\mathrm{O}}>.38\right.$ ) indicates the same shape (within the statistical errors and those of the stability of the electronics) for metallic iron, $\mathrm{Fe}_{2} \mathrm{O}_{3}$ and $\mathrm{Fe}_{3} \mathrm{O}_{4}$. Similar comparisons of metals and oxides beyond the oxygen endpoint for $\mathrm{Mg}, \mathrm{Al}$ and $\mathrm{Si}$, likewise show agreement, with the structure in the scattering spectra being preserved (see below, Figure 14).

A more severe test of the independence of the shape of the spectrum on the chemical composition lies in the region of 


\section{FIGURE 14}

Separation of the Alpha Scattering Spectrum from Mgo into $\mathrm{Mg}$ and $\mathrm{O}$ components. The data were obtained with the Research Apparatus. The vertical lines associated with each point represent statistical errors.

$$
\begin{aligned}
& \text { original data from MgO in units of } \\
& \text { counts per } 4 \text { channels per } 100 \text { min. } \\
& \text { data from Mg metal fitted to the Mgo } \\
& \text { data beyond channel } 72 \text {. The } \\
& \text { normalization factor is } 0.494 \pm 0.024 . \\
& \text { Contribution due to oxygen based on } \\
& \text { subtraction of Mg data from that of } \\
& \text { Mgo. The slight peak in the oxygen } \\
& \text { curve at around channel } 60 \text { may be due } \\
& \text { to instrument gain shifts between the } \\
& \text { time of measurement of the Mgo and Mg } \\
& \text { samples. This is indicated by the } \\
& \text { slight peak shift of the Mg component } \\
& \text { at channel } 85 \text {. }
\end{aligned}
$$




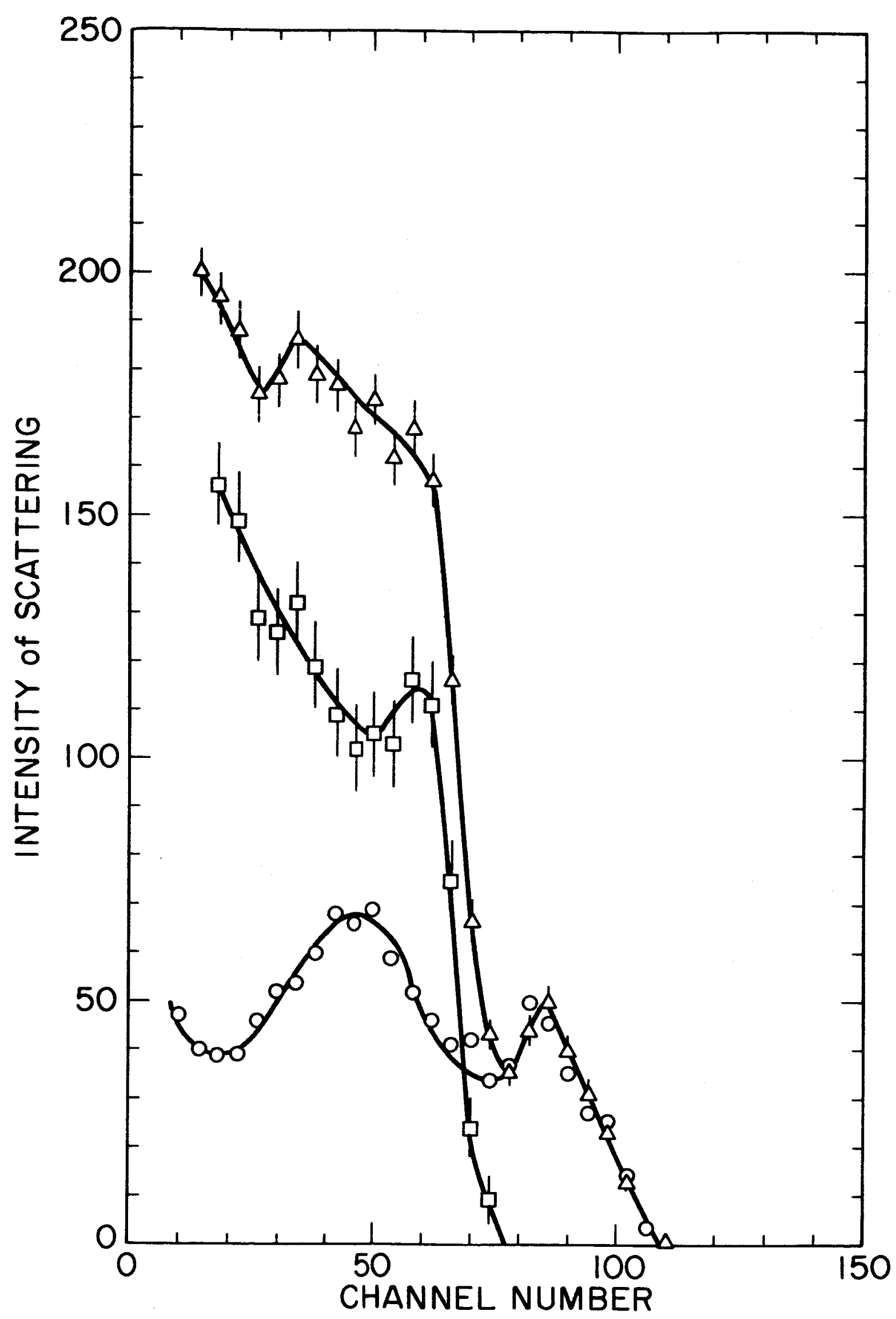


low energies since the approximations leading to Equation 9 are less certain at low energies. As an example of this situation, the oxygen contribution was obtained from the oxides of $\mathrm{Mg}$ and two oxides of $\mathrm{Fe}$ by subtracting the metal contribution assuming it was the same at low energies as beyond the oxygen endpoint. The procedure is illustrated in Figure 14 for the case of magnesium-magnesium oxide. This figure shows the intensity of scattering from $\mathrm{MgO}$ in the Research Apparatus, as a function of energy (channel number). Beyond channel 72, the spectrum is representable by $(0.494 \pm 0.024)$ times the magnesium metal spectrum in the same apparatus. Subtraction of the metal spectrum multiplied by 0.494 from the oxide spectrum gives the contribution of oxygen to the oxide spectrum.

The oxygen contributions obtained in this manner from $\mathrm{MgO}, \mathrm{Fe}_{2} \mathrm{O}_{3}$ and $\mathrm{Fe}_{3} \mathrm{O}_{4}$ are shown in Figure 15 on a semilogarithmic plot. It is seen that the shapes of the derived curves are very similar. The slight peak at about channel number 60 in the oxygen curve from Mgo may be real, or it may be the result of a slight amplification shift in the equipment in between the time of measurement of the $\mathrm{MgO}$ and of the $\mathrm{Mg}$. For the present discussion it would appear as if, within statistical and instrumental drift errors, the shapes of the oxygen contributions from the three oxides are the same. 


\section{FIGURE 15}

The shape of the alpha scattering spectrum due to oxygen as obtained from several oxides. The data were obtained on the Research Apparatus. The ordinate is the intensity in units of counts per 4 channels per 100 min. on a logarithmic scale; the abscissas are channel numbers (proportional to energy). The various types of points were obtained from different oxide - metal data in the manner illustrated for $\mathrm{Mg}$ (see Figure 14). The statistical error bars have been shown only in the case of the $\mathrm{MgO}-\mathrm{Mg}$ points. The absolute intensities have not been corrected for differences in the ionization energy losses in the various systems. 


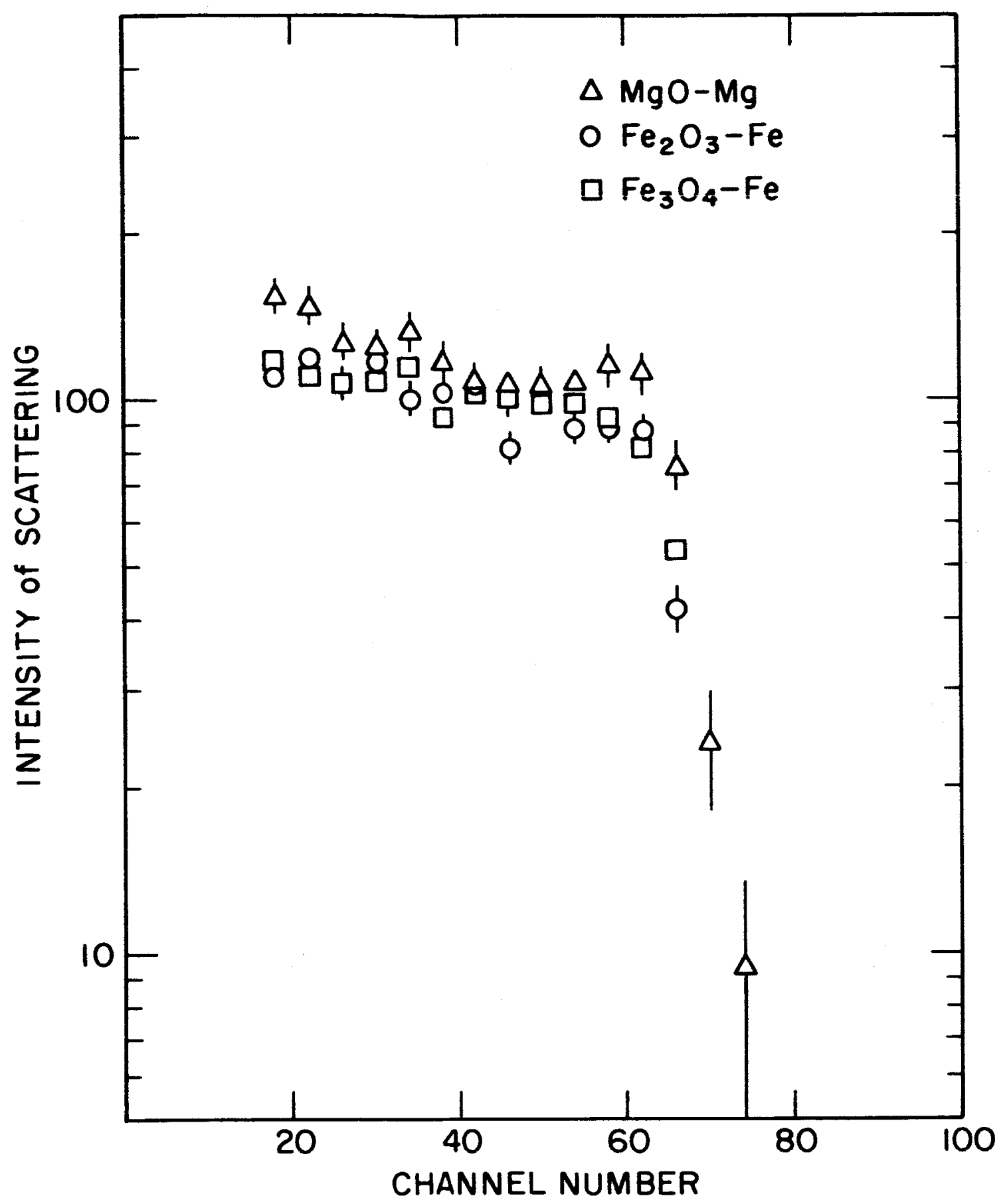


If it is now assumed that the shapes of the scattered spectra are independent of the chemical state, the intensities can be compared with predictions from ionization energy loss considerations. According to these, the intensities of an element in different compounds will be proportional to the atomic fraction of the element divided by the average ionization energy loss per atom of the substance being investigated (see Equation 10):

$$
\frac{d I_{i}}{d T_{3}}=\text { const } \frac{x_{i}}{\bar{\varphi}}
$$

The simplest assumption about the ionization energy losses per atom, $\varphi_{i}$, is that they vary as $\sqrt{z_{i}}$, or, what is equivalent for light elements, as $\sqrt{A_{i}}$. Figure 16 illustrates the extent to which this relation is obeyed in the case of carbon, oxygen, magnesium, silicon and iron in various chemical states. It is seen that the proportionality of intensity to $\left.\frac{x_{i}}{\left\langle\sqrt{A_{i}}\right.}\right\rangle$ is followed moderately well for $\mathrm{Fe}, \mathrm{Mg}$, Si and $\mathrm{O}$, but only to about 15-20\% for carbon. These larger deviations for carbon may be associated with the failure of the simplified ionization loss assumptions for a light element.

Using these assumptions, of the independence of composition of the scattering spectral shape of an element and the $\sqrt{\mathrm{A}_{i}}$ dependence of the ionization cross section per atom, 
FIGURE 16

Dependence of the intensity of scattering by an element on concentration. Data are presented for the intensity of scattering by carbon, oxygen, magnesium, silicon and iron in different substances. The abscissas are the atomic fraction of the element in the system divided by the average square root of the mass number of the system, $\mathrm{x}_{i} /<\sqrt{\mathrm{A}}>$; the ordinates are intensities of scattering by the element at a given energy in units of counts per 100 min. per 4 channels at a source strength of $10^{10} \mathrm{~d} / \mathrm{m}$. The carbon data are from samples of $\mathrm{CaCO}_{3}$, polyethylene, $\mathrm{B}_{4} \mathrm{C}$, and graphite (the coordinates in the case of graphite and polyethylene have both been divided by 4). The oxygen data are from $\mathrm{Fe}_{2} \mathrm{O}_{3}, \mathrm{MgO}_{2} \mathrm{Al}_{2} \mathrm{O}_{3}, \mathrm{TiO}_{2}, \mathrm{CaCO}_{3}$ and $\mathrm{SiO}_{2}$. The data for magnesium were obtained using the substances $\mathrm{Mg}_{3}\left(\mathrm{PO}_{4}\right)_{2}, \mathrm{MgSO}_{4}, \mathrm{MgO}$ and magnesium metal. The silicon data are obtained from $\mathrm{SiO}_{2}$ and elementary silicon. $\mathrm{Fe}_{3} \mathrm{O}_{4}, \mathrm{Fe}_{2} \mathrm{O}_{3}$ and iron metal were used to obtain the iron data. 


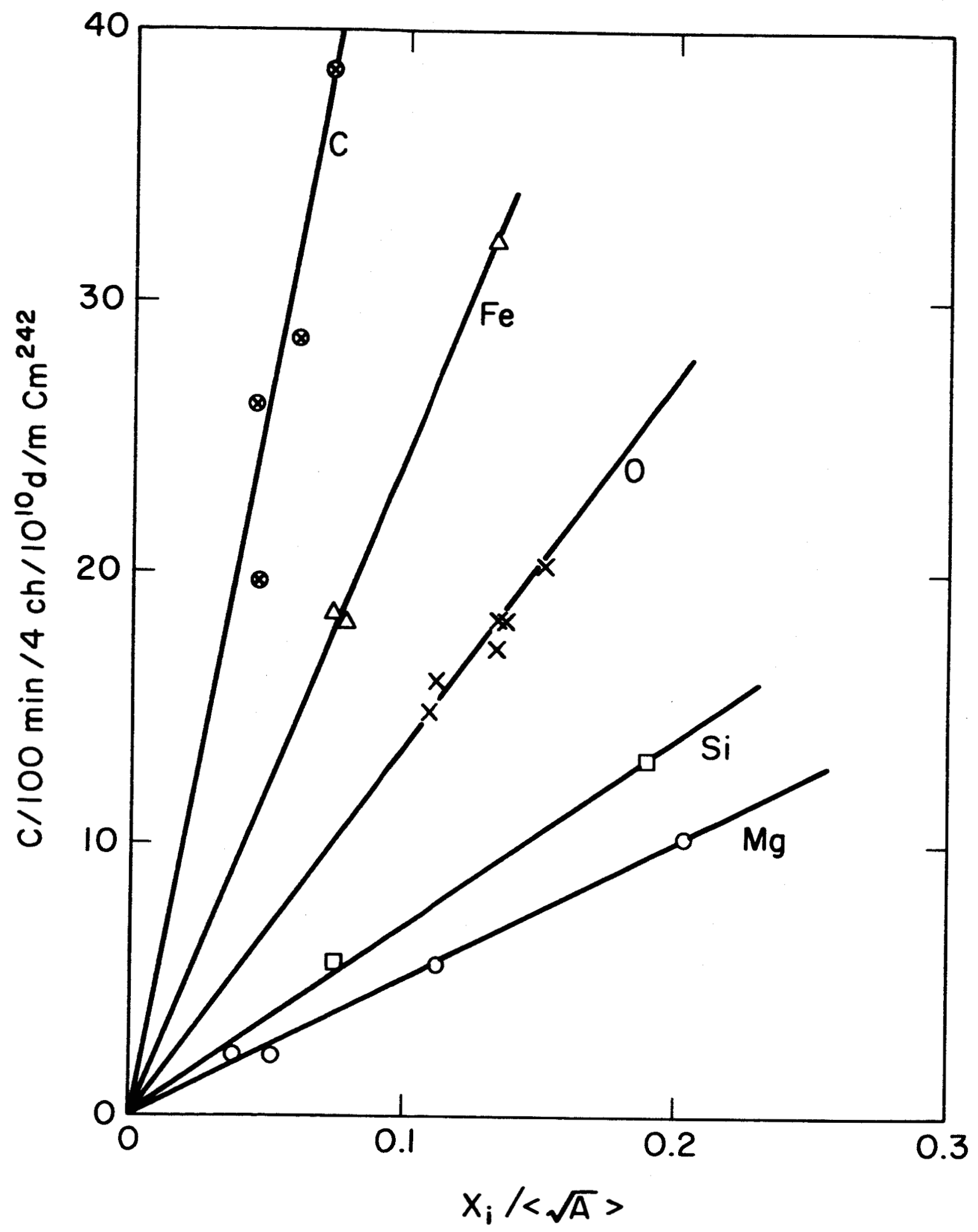


the scattering spectra of pure elements not easily available in elementary form can be obtained from those of compounds. The resulting curves for sodium, chlorine, and calcium are shown in Figure 17. In this manner a library of alpha scattering spectra applicable for a particular instrument can be obtained experimentaliy for all elements of interest. For heavy elements for which the scattering is predictable theoretically, approximate curves can be obtained for elements not investigated by interpolation between measured spectra.

The theoretical considerations about the superpositions and intensities of the scattered alpha particle spectra are expected to be even more valid in the case of the proton spectra from $(\alpha, p)$ reactions. This follows from the higher velocities of protons than of alpha particles of the same energy. Because of this, the ionization energy losses of protons as a function of energy are more rigorously only multiplicatively dependent on the nature of the target material. Moreover, the $(\alpha, p)$ reactions occur in a restricted energy range, in which the dependence of the ionization loss cross section per atom for alpha particles does vary as the square root of the mass number. These considerations are tested in Figure 18. In this figure the energy integrated proton intensity from several compounds having one proton producing element is plotted as a function of the fraction of proton producing nuclei divided by the average square root of the mass number of the medium. 


\section{FIGURE 17}

Alpha scattering spectrums for Sodium, Chlorine and Calcium. These spectrums have been deduced from compounds containing these elements by the methods of the discussion section. The sodium spectrum is derived from the spectrum of sodium fluoride; that of chlorine is derived from the spectrum of potassium chloride; the spectrum of calcium is obtained from that of calcium oxide. 


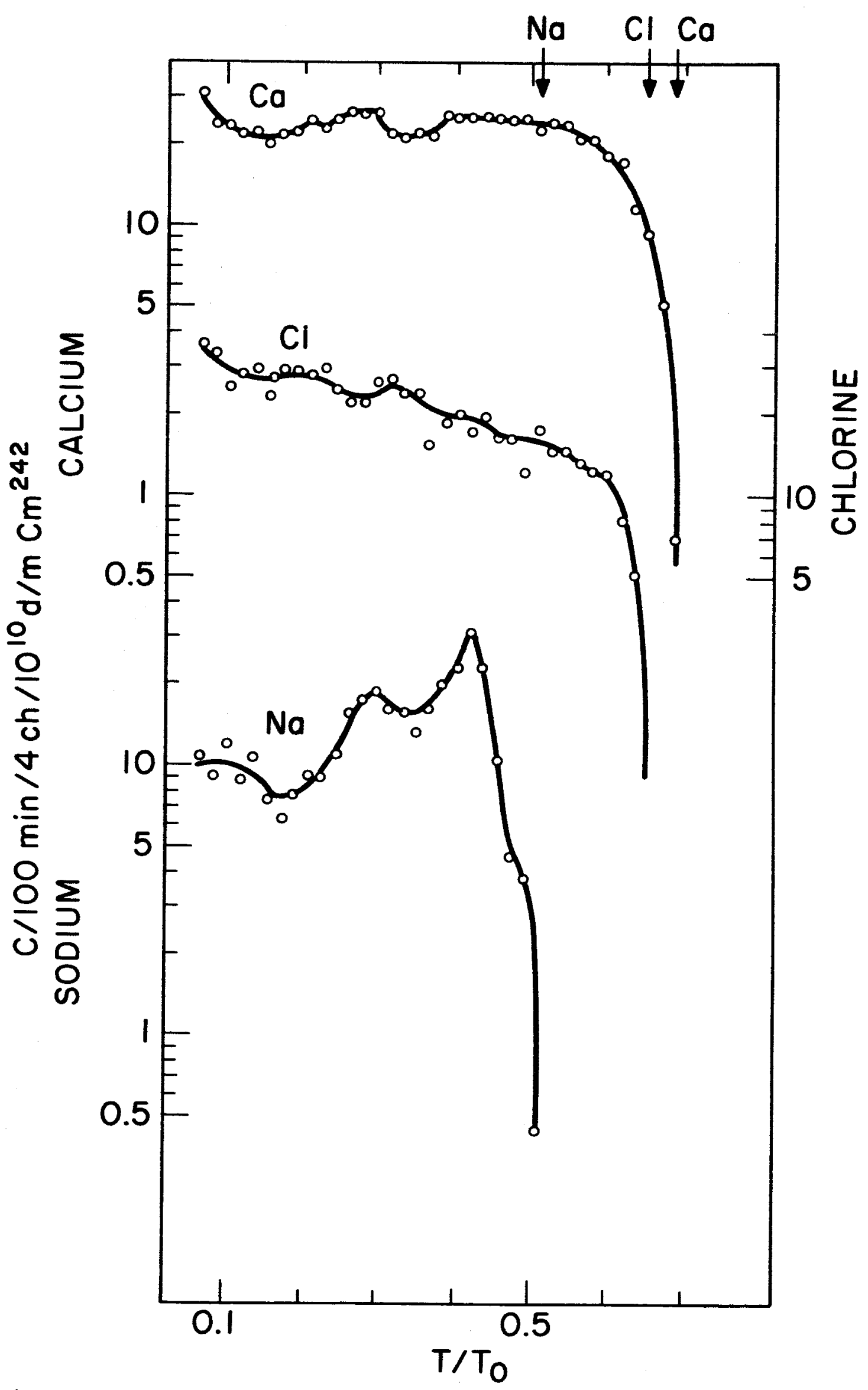


Data are presented for sodium, aluminum and silicon. The proportionality to this function of concentration is seen to be adequately obeyed for all three elements.

In these tests of the quantitative aspects of analysis with alpha particles the weighted square root of the mass number has been used in abscissas in Figure 17 and 18. In a sample of unknown composition this will be unknown. However, this function is the same for all components of the same material and so the relative amounts of the different elements will be determined from the relative intensities of the different components compared to those of the pure elements.

The theoretical considerations presented above apply strictly only to targets that are chemically homogeneous. Since alpha particles of the energy used in this work have ranges of the order of $5-10 \mathrm{mg} / \mathrm{cm}^{2}$, this homogeneity must be to a depth of half this amount or of the order of 10 microns for typical materials. Similarly the considerations apply only to material that is homogeneous laterally across the face of the target. In the case of heterogeneous materials the gross scattering and proton spectra will be appropriate averages .

The effect of surface smoothness on the spectral shape of scattered alpha particles has received some attention (Eskind and Mark, 1962). From geometrical considerations it 
FIGURE 18

Dependence of proton intensity from $(\alpha, p)$ reactions on concentration of proton producing element. Data are presented for the integrated intensity of protons from sodium, aluminum and silicon in different substances. The abscissas are the atomic fraction of the element in the system divided by the average square root of the mass number of the system, $x_{i} /<\sqrt{A}>$; the ordinates are arbitrary intensity units. The sodium data are from samples $\mathrm{NaCl}$ and $\mathrm{Na}_{2} \mathrm{CO}_{3}$; the aluminum and silicon data are from the metal and the oxide. The small contribution of protons from chlorine has not been subtracted from the $\mathrm{NaCl}$ data. 


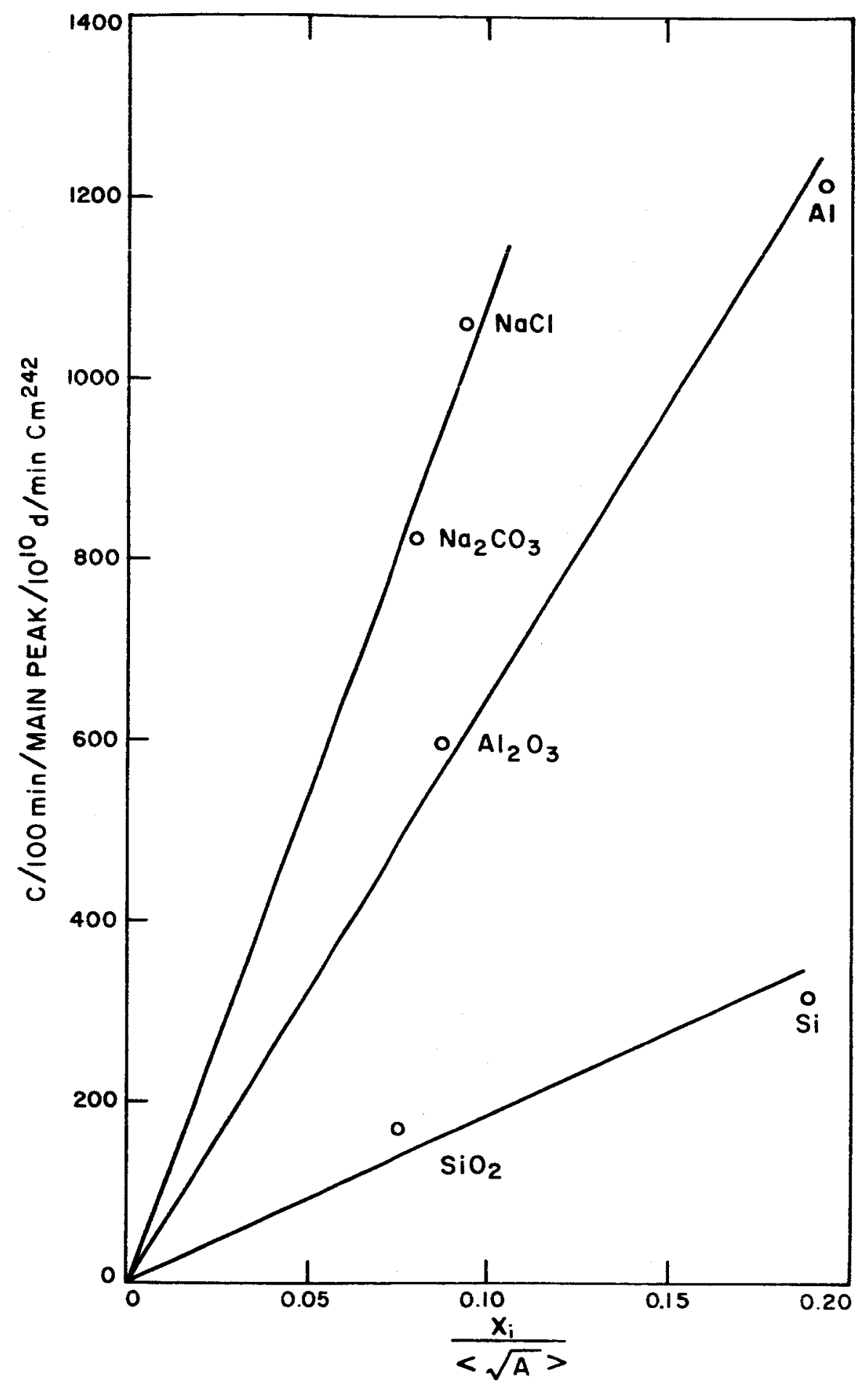


is clear that, as the scattering angle approaches $180^{\circ}$, surface irregularities, even on a micron scale, should have no effect. Under the conditions used in practical instruments there has been found no effect on the shapes of the spectra in comparing the spectra of polished metals and finely powdered oxides. In addition, a series of experiments have been performed using $\mathrm{Al}_{2} \mathrm{O}_{3}$ powders of various sizes (.05-20 microns). The oxygen contribution in the different samples was obtained in a standard fashion, and was indistinguishable visually. A least squares fit of the data in the region of the oxygen endpoint was made to the function proposed by Eskind and Mark (1962).

$$
I(c)=\frac{k}{e^{a\left(c-c_{0}\right)}+I}
$$

where $I(c)$ is the intensity of scattering measured in a channel $c, c_{0}$ is the channel in which the intensity falls to half its plateau value, $k$ is a normalization constant, and a determines the shape of the spectrum near $c_{0}$. The values of a deduced for the various samples are presented in Table 3. Within the statistical errors they are constant and show no trend with particle size. A similarly rigorous treatment of the aluminum portion of the scattering spectra could not be made because of the structure of the spectra and because the samples had varying amounts of higher $z$ impurities. 
TABLE 3. Effect of Particle Size on Shape of Alpha Scattered Spectrum*

\begin{tabular}{cl}
\hline $\begin{array}{l}\text { Average particle } \\
\text { size of } \mathrm{Al}_{2} \mathrm{O}_{3}\end{array}$ & $\begin{array}{l}\text { least squares fit } \\
\text { to parameter, a, } \\
\text { powder (microns) }\end{array}$ \\
\hline 20 & $0.20 \pm 0.06$ \\
5 & $0.15 \pm 0.05$ \\
0.3 & $0.16 \pm 0.05$ \\
0.05 & $0.19 \pm 0.04$ \\
\hline
\end{tabular}

* These data were obtained with an average scattering angle of $160^{\circ}$. 
The optimum application of the alpha scattering technique to the analysis of complex mixtures obviously requires the acquisition of a library of alpha and proton spectra obtained under identical geometrical and electronic conditions. A complex spectrum is then analyzed either by successively peeling off the components containing the highest energy particles, or by separating the complex spectrum into components using a computer.

As an illustration of this technique Figure 19 presents the alpha scattering spectrum (A) and proton spectrum (B) obtained from plate glass in the breadboard instrument. Figure 20 shows the result of decomposition of the spectrum using the $\sqrt{\mathrm{A}}$ ionization energy loss relationship. Presented are the results of the analysis using only the alpha spectrum, only the proton spectrum, and using the results of both. Also are shown the results of a spectrographic analysis of the plateglass (oxygen by difference).

Figure 20 shows that the alpha scattering spectrum determines the most abundant elements - oxygen and silicon directly, and moderately well. Likewise the calcium is determined adequately by the alpha particles. The alpha spectrum does not provide good discrimination between $\mathrm{Na}$, Mg and Al. Use of the information from the proton spectra is a significant help here, as expected. 


\title{
FIGURE 19
}

\begin{abstract}
Alpha scattering spectrum (A) and proton spectrum (B) obtained in the Breadboard Instrument from a sample of plate glass. Calculated high energy endpoints for major components of the glass are shown for the alpha scattering spectrum; prominent features of the proton spectrum due to $\mathrm{Si}$ and $\mathrm{Na}$ are indicated. The spectra obtained with crushed glass were the same within statistics as with a sheet of glass.
\end{abstract}




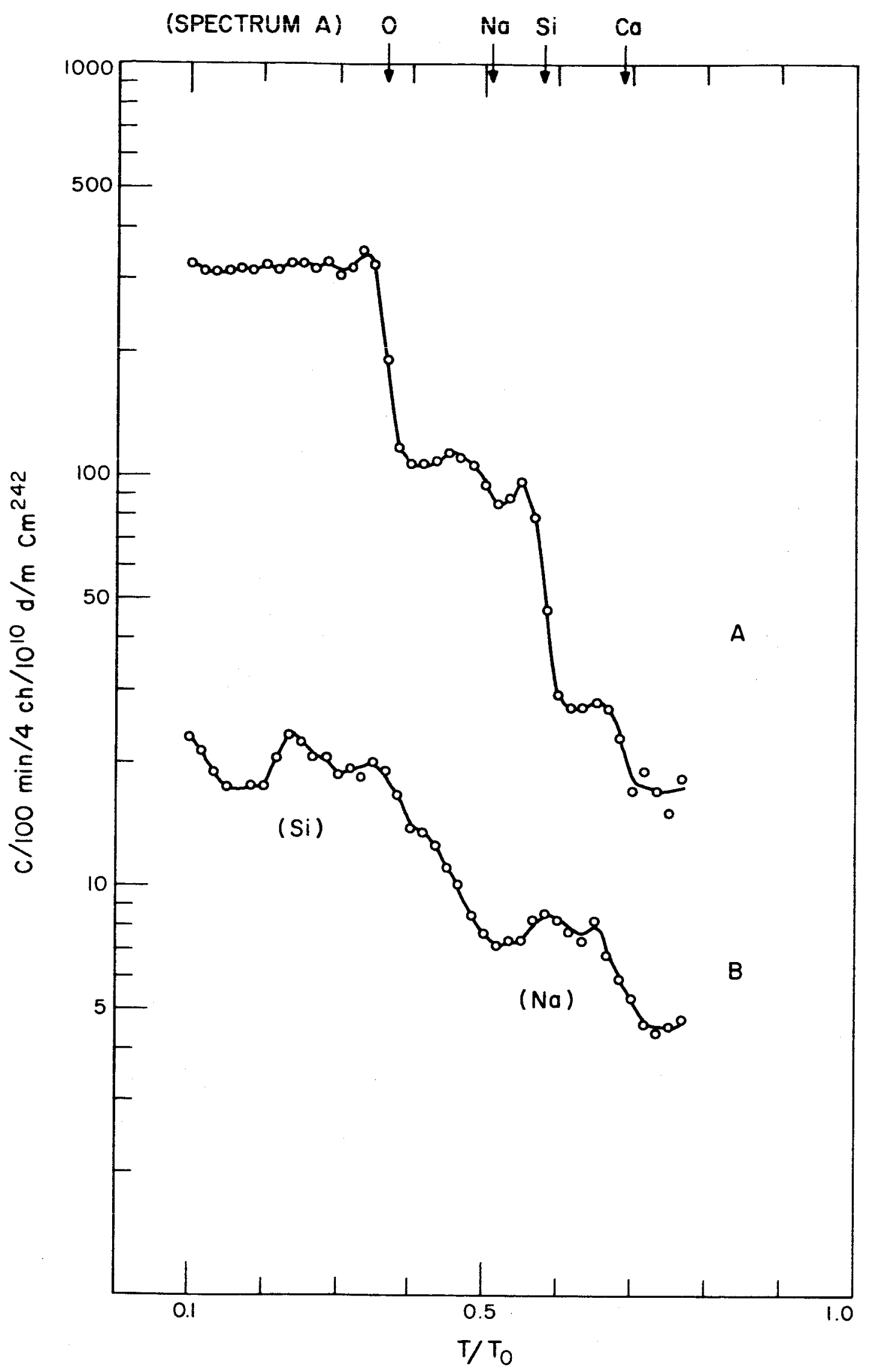




\section{FIGURE 20}

Comparison of least-square computer analyses of alpha scattering and proton spectra of plate glass with a conventional analysis. The proton analysis includes only the proton-producing elements $\mathrm{Na}, \mathrm{Mg}, \mathrm{Al}$, and $\mathrm{Si}$. The conventional analysis gives oxygen by difference. 

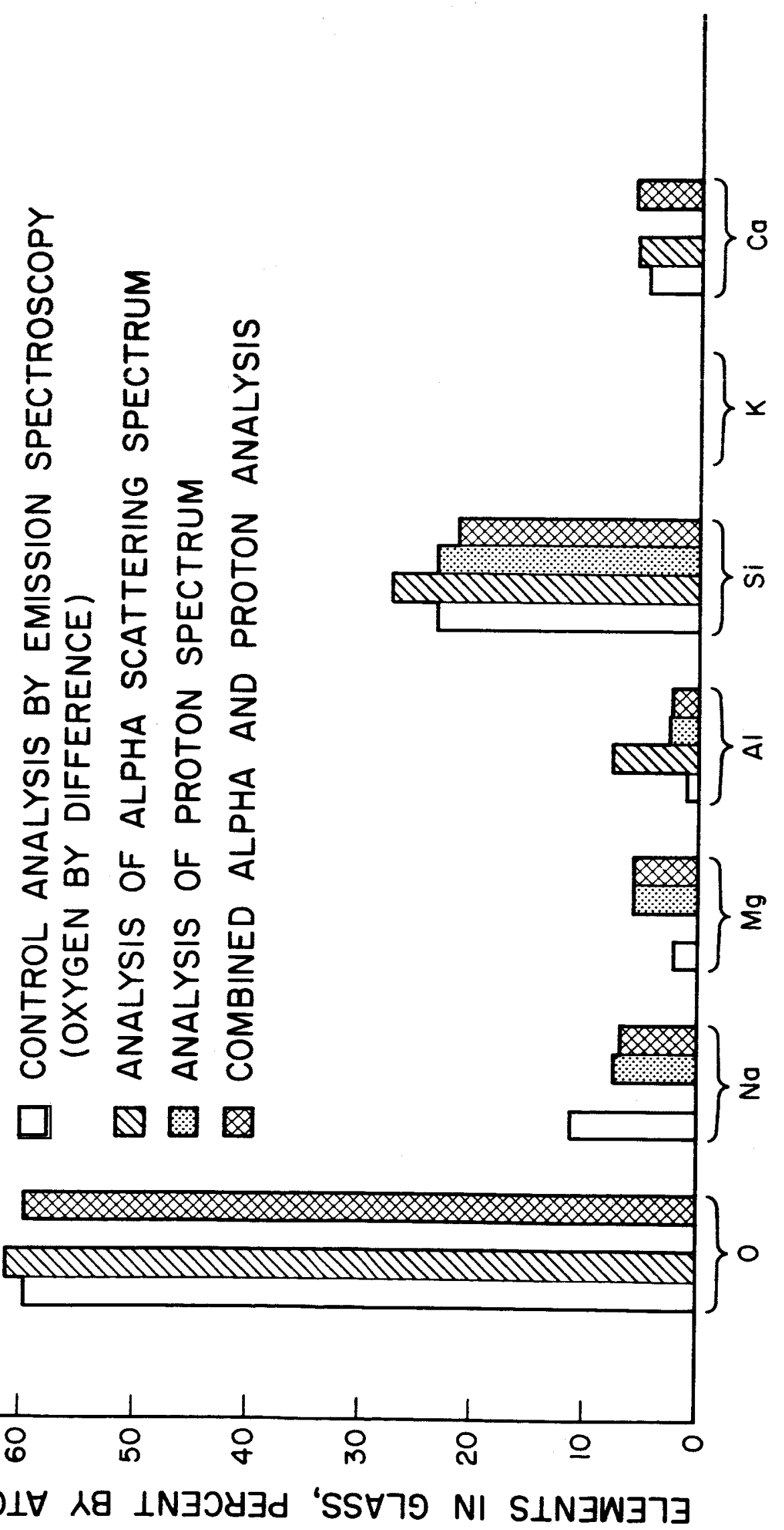
Although the work to date on complex mixtures has been limited in scope, it would appear to support the expectation that this technique could give information on the elemental composition of surfaces of materials. The simplicity of the equipment would seem to recommend it for instmumted space missions.

\section{ACKNOWLEDGEMENT}

The authors acknowledge the support of the following colleagues in this work: The members of the chemistry Division of the Lawrence Radiation Laboratory, Livermore, California, for samples of neutron irradiated $\mathrm{Am}^{24 I}$; Dr. T. Hincks of the National Research Council of Canada and Dr. H. L. Anderson of the Enrico Fermi Institute for Nuclear Studies of the University of Chicago for the first charge sensitive amplifiers and Dr. Robert Takaki, Mr. Blaine Arneson and Mr. James Lamport of the Chicago Laboratory for Applied Sciences for later transistorized amplifiers and pulse height analyzers used in the breadboard and prototype instruments; Dr. A. Tuzzolino and Dr. J. Kristoff of the same laboratory for the preparation of many special detectors; Mr. Dale J. Henderson of the Argonne National Laboratory for electronic assistance and all the alpha and gamma pulse analyses needed in the preparation of sources; Mr. E. Blume and Mr. A. Beretvas of the Enrico Fermi Institute for Nuclear Studies of the University of Chicago for the mechanical design of the instruments and much general assistance. 


\section{REFERENCES}

* The work at the Argonne National Laboratory was performed under the auspices of the United States Atomic Energy Commission.

* The work at the Enrico Fermi Institute for Nuclear Studies was supported by Grant NsG-127-6I of the National Aeronautics and Space Administration.

Bittner, J.W., and R. D. Moffat.

Elastic Scattering of Alpha Particles by Carbon Phys. Rev. 96, 374 (1954).

Eskind, Neil A., and H. Mark.

Mesh Size Effects in Alpha Particle Backscattering

J. Geophys. Res. 67, 4867 (1962) .

Ferguson, A. J., and L. R. Walker.

The Scattering of Alpha Particles by Carbon and Oxygen Phys. Rev. 58, 666 (1940).

Friedlander, G., and J.W. Kennedy.

Nuclear and Radiochemistry

John Wiley and Sons, 1955, P.192.

Rutherford, E., J. Chadwick, and C. D. Ellis.

Radiations from Radioactive Substances

Cambridge University Press, 1930, P.244. 
Turkevich, Anthony.

Chemical Analysis of Surfaces by Use of Large Angle

Scattering of Heavy Charged Particles

Science, 134, 672 (1961).

Whaling, Ward.

The Energy Loss of Charged Particles in Matter

Handbuch der Physih, 34, 193, Springer Verlag, Berlin, 1958. 